\title{
Intramembrane protease RHBDL4 interacts with erlin complex to target unstable soluble proteins for degradation
}

Nathalie Kühnle ${ }^{1,3}$, Josephine Bock ${ }^{1,3}$, Julia D. Knopf ${ }^{1}$, Nina Landscheidt ${ }^{1}$, Jin-Gu Lee ${ }^{2}$, Yihong $\mathrm{Ye}^{2}$ and Marius K. Lemberg ${ }^{1, *}$

${ }^{1}$ Center for Molecular Biology of Heidelberg University (ZMBH), DKFZ-ZMBH Alliance, Im Neuenheimer Feld 282, 69120 Heidelberg, Germany.

${ }^{2}$ Laboratory of Molecular Biology, National Institutes of Health, Bethesda, MD 20892, USA.

${ }^{3}$ These authors contributed equally to this work.

${ }^{*}$ Corresponding author: m.lemberg@zmbh.uni-heidelberg.de

Running title: Pre-aggregate clearance by RHBDL4

Keywords: intramembrane proteolysis, ER-associated protein degradation, prohibitin family proteins Erlin1 and Erlin2, insoluble protein aggregates

\begin{abstract}
Protein degradation is fundamentally important to ensure cell homeostasis. In the Endoplasmic Reticulum (ER), the ER-associated degradation (ERAD) pathway targets incorrectly folded and unassembled proteins into the cytoplasm for turnover by the proteasome. In contrast, lysosomal degradation serves as failsafe mechanism for removal of proteins that resist ERAD by forming aggregates. In previous work, we showed that the ERresident rhomboid protease RHBDL4, together with $\mathrm{p} 97$, mediates membrane protein degradation. However, whether RHBDL4 acts in concert with additional ERAD components is unclear and its full substrate spectrum remains to be defined. Here, we show that besides membrane proteins, RHBDL4 cleaves aggregation-prone, luminal ERAD substrates including a soluble version of the major histocompatibility complex heavy chain (MHC202). RHBDL4's interaction with erlin ERAD substrate receptors and reciprocal interaction of MHC202 with erlins suggest that RHBDL4 defines a substrate clipping mechanism that rescues aggregation-prone peptides in the ER lumen from terminal aggregation.
\end{abstract}

\section{Abbreviations}

ER, endoplasmic reticulum; ERAD, ER-associated degradation; MHC, major histocompatibility complex; TM, transmembrane; UPR, unfolded protein response. 


\section{Introduction}

Around one third of all proteins enter the secretory pathway through the endoplasmic reticulum (ER), turning it into a crowded folding compartment. Even though numerous factors assist folding and complex assembly, this is an error prone process and misfolded polypeptides or orphan complex subunits arise, that are commonly removed by the ERassociated degradation (ERAD) pathway (Christianson and Ye, 2014; Juszkiewicz and Hegde, 2018; Ruggiano et al., 2014; Wu and Rapoport, 2018). If the burden of misfolded proteins exceeds the capacity of the protein homeostasis (proteostasis) network, aggregation-prone polypeptides form clusters. Depending on the protein, these clusters consists of unstructured, amorphous aggregates or structured $\beta$-sheet amyloid fibers (Balchin et al., 2016; Breydo and Uversky, 2015). Protein aggregates cause cellular toxicity and are a hallmark of several diseases including neurodegenerative disorders, like Alzheimer's or Parkinson's disease (Chiti and Dobson, 2017). Clearance of large misfolded protein species in the ER is accomplished by selective autophagy (Grumati et al., 2018; Kruse et al., 2006a) or a recently described vesicular ER-to-lysosome trafficking pathway (Fregno et al., 2018). Yet, if not terminally aggregated, the best characterized mechanism for turnover of aberrant proteins is ERAD. Here, as part of the canonical ER quality control, misfolded proteins are recognized by a network of protein factors including chaperones, glycan-modifying enzymes and protein disulfide isomerases and reductases (Christianson et al., 2011).

ERAD consists of several parallel pathways that allow removal of a quite diverse set of aberrant proteins. Best understood in yeast, three major degradation routes, namely ERADL, ERAD-M and ERAD-C, are formed by distinct E3 ubiquitin ligase complexes that recognize proteins with lesions in the lumen, ER membrane or cytoplasm, respectively (Carvalho et al., 2006; Denic et al., 2006). Although this distinction may not be as strict in mammalian cells, a defined set of quality control factors still assists turnover of different protein classes (Bernasconi et al., 2010; Christianson et al., 2011). Glycosylated ERAD-L substrates often engage the lectins calnexin and calreticulin, $\alpha 1$-mannosidases (EDEM1, -2 and 3) and the disulfide reductase ERdj5 that collectively routes proteins via Sel1 to the E3 ubiquitin ligase Hrd1 (McCaffrey and Braakman, 2016; Ruggiano et al., 2014). Moreover, for turnover of soluble ERAD substrates frequently catalytically-inactive rhomboid protease homologues referred to as pseudoproteases (Der1 in yeast and Derlin1, -2 and -3 in humans) are required (Christianson et al., 2011; Greenblatt et al., 2011; Vashist and Ng, 2004). After recruitment by a E3 ubiquitin ligase complex containing a derlin protein, ERAD-L substrates are dislocated across the ER membrane to reach the proteasome $(\mathrm{Wu}$ and Rapoport, 2018). Once emerged into the cytosol, ERAD substrates are commonly extracted 
by the AAA+-ATPase p97 (Cdc48 in yeast), deglycosylated by an N-glycanase and finally degraded by the proteasome (Hirsch et al., 2003; Ye et al., 2001). Work in yeast and in vitro suggest that for ERAD-L substrates Hrd1 forms the core of a dislocation channel together with the Sel1 orthologue Hrd3 (Baldridge and Rapoport, 2016; Schoebel et al., 2017). However, alternative ERAD pathways exist, as for example degradation of activated inositol 1,4,5-triphosphate IP(3) receptors engages a Mega-Dalton (MDa) complex consisting of multiple copies of the type II membrane proteins Erlin1 and -2 and the E3 ubiquitin ligase RNF170 (Lu et al., 2011; Pearce et al., 2007; Pearce et al., 2009). As a variation to the theme, several ERAD substrates are processed by intramembrane proteases prior extraction from the ER membrane (Avci and Lemberg, 2015). Accordingly, the rhomboid intramembrane protease RHBDL4 has been linked to ERAD (Fleig et al., 2012; Paschkowsky et al., 2018) that impacts key aspects of the secretory pathway such as tuning the N-linked glycosylation machinery and the rate of ER export (Knopf et al., submitted; Wunderle et al., 2016). RHBDL4 uses a bipartite substrate recognition mechanism to select certain membrane proteins with unstable TM domains. Primarily, RHBDL4 recognizes positively charged residues within TM domains (Fleig et al., 2012; Paschkowsky et al., 2018), which destabilize the TM helix and act as degradation signals (degron) of ERAD-M substrates (Bonifacino et al., 1990). As a second layer of control, substrate recognition occurs through a conserved ubiquitin-interacting motif at the cytosolic C-terminal tail of RHBDL4 (Fleig et al., 2012). Therefore, RHBDL4 does not solely rely on one recognition mechanism. Rather, it integrates different information including substrate ubiquitination before it performs the irreversible action of cleavage. What features determine whether a protein enters a classical ERAD pathway or is first cleaved by RHBDL4 or another ER protease are unknown.

By asking what influence different proteostasis factors have on turnover of ERAD-L substrates, we discovered that in addition to its role in ERAD-M RHBDL4 serves as a noncanonical factor in clearance of misfolded soluble proteins in the ER lumen. This shows that the substrate spectrum of rhomboid intramembrane proteases is more diverse than originally anticipated. Moreover, we demonstrate that for clearance of luminal substrates, RHBDL4 cooperates with the erlin complex, a putative ERAD recruitment factor for aggregation-prone peptides. Since RHBDL4 ablation increases the load of insoluble versions of its substrates, we suggest that the RHBDL4-erlin complex plays an important role in pre-aggregate clearance from the ER lumen via dislocation into the cytoplasm and proteasomal degradation. 


\section{Results}

A targeted siRNA screen identifies RHBDL4 as ERAD-L component

To investigate principles of ERAD pathway selection, we transfected a soluble model ERAD substrate into Hek293T cells and analyzed its steady-state level in an siRNA screen. As model substrate we generated a truncated version of the major histocompatibility complex (MHC) class I heavy chain of 202 amino acids (MHC202), which comprises an antiparallel $\beta$ sheet and two $\alpha$-helices formed by a tandem repeat of the so-called $\alpha 1$ and $\alpha 2$ domains (Figure 1A). Based on the primary sequence and crystal structure of the MHC ectodomain (Bulek et al., 2012), we predicted that the soluble MHC202 truncation forms an unstable protein containing one $\mathrm{N}$-linked glycan and one disulfide bridge that exposes an extensive hydrophobic surface (Figure 1A, bottom panel right). For cell-based screening, we tested p97 that is invariant for protein dislocation and 40 proteins that have been implicated in ERAD either by acting as an E3 ubiquitin ligase or by participating in the reported ERAD protein interacting network (Christianson et al., 2011; Christianson and Ye, 2014). With a threshold of three-fold enrichment, 11 candidates showed an effect of which p97 and the E3 ligase Hrd1 showed the strongest MHC202 steady state increase (Figure 1B and S1A). Moreover, knockdown of the Hrd1-associated ERAD factors Herp, Derlin2/3, Sel1, the $\alpha 1$ mannosidases EDEM1/2, and the disulfide reductase Erdj5, caused a robust increase of MHC202 (Figure 1B and S1A). However, knockdown of the lectin OS9, which typically targets glycoprotein ERAD substrates to Sel1 did not alter MHC202 levels (Figure 1B and $\mathrm{S} 1 \mathrm{~A}$ ), indicating a redundant function of the paralogue XTP-3B that has been observed for certain other substrates (van der Goot et al., 2018). While all these factors are known to be required for recognition and degradation of glycosylated ERAD-L substrates (Christianson et al., 2011), we also observed that knockdown of the putative membrane-integral ER quality control factor Bap29 (Abe et al., 2009) and the rhomboid intramembrane protease RHBDL4 (Fleig et al., 2012) caused a subtle increase of MHC202. These factors had not been linked to Hrd1-Sel1 (Christianson et al., 2011), indicating that also non-canonical factors contribute to MHC202-clearance.

Intramembrane proteases are commonly believed to cleave only membrane-integral proteins but exceptions are known (Kühnle et al., 2019). We therefore set out to characterize the unexpected role of RHBDL4 in MHC202 turnover. First, we confirmed that knockdown with two independent targeting sequences elevated MHC202 steady-state levels (Figure S1B). The role of RHBDL4 in MHC202 turnover was further confirmed by cycloheximide chase in RHBDL4 knockout Hek293T cells (Figure S1C), in which the half-live of MHC202 turnover increased from less than one hour to approx. two hours (Figure 1C). However, inhibition was only partial, indicating that more than one pathway targets MHC202 for degradation. Hence, 
we presume that induction of the ER unfolded protein response (UPR) observed upon RHBDL4 ablation (Fleig et al., 2012) masks the RHBDL4 knockout phenotype to a certain extent by upregulating alternative degradation routes. Consistent with this, short-term knockdown of Hrd1 and RHBDL4 that does not to induce the UPR slightly increased MHC202 steady state levels while under these conditions double knockdown leads to additive effects (Figure S1D and data not shown). Next, we asked, whether RHBDL4 directly processes MHC202 by performing a cell-based rhomboid gain-of-function cleavage assay (Fleig et al., 2012). Consistent with such a direct role of rhomboid-catalyzed cleavage in MHC202 clearance, overexpression of wild-type RHBDL4 (wt) but not its catalytic serine144-alanine mutant (RHBDL4-SA) generated an N-terminal fragment with an apparent molecular weight of $18 \mathrm{kDa}$ (Figure 1D). While only traces of this cleavage product were observed in vehicle-treated cells, inhibition of the proteasome with MG132 increased its steady-state level. This result indicates that RHBDL4 generates a MHC202-cleavage fragment which is dislocated into the cytoplasm for proteasomal degradation in an analogues manner as previously described for membrane-integral substrates (Fleig et al., 2012). Likewise, proteasome inhibition also stabilized deglycosylated full-length MHC202 (Figure 1D and S1E). Again, this shows that MHC202 is degraded by both, the canonical Hrd1 dislocation route and a RHBDL4-dependent substrate clipping mechanism (Avci and Lemberg, 2015). Consequently, the full extent of RHBDL4 activity can only be seen when the downstream clearance pathway for fragments is blocked. Interestingly, overexpression of the catalytic inactive SA mutant stabilized the deglycosylated form of MHC202 even in absence of MG132. This observation suggests that in a dominant negative manner the RHBDL4-SA mutant traps a partially dislocated form of MHC202 exposing the glycosylation site to the cytoplasmic N-glycanase, while MHC202 is bound to the rhomboid active site. Consistent with this model, in a co-immunoprecipitation assay from Triton X-100-solubilized cells, RHBDL4-SA showed physical interaction with the glycosylated as well the deglycosylated MHC202 species, whereas ectopically expressed FLAG-tagged OS9 was not bound (Figure $1 \mathrm{E})$. Taken together, these results indicate that RHBDL4 physically interacts with dislocating MHC202 thereby generating cleavage fragments that are released into the cytoplasm where they become degraded by the proteasome.

\section{RHBDL4 cleaves selected soluble ERAD-L substrates}

Next, we asked whether also other soluble ERAD-L substrates are cleaved in the cell-based RHBDL4 cleavage assay. Neither, the null Hong Kong mutant of $\alpha 1$-antitrypsin (NHK) (Hosokawa et al., 2003) nor an ER-retained mutant of prolactin (Prl-KDEL) (Fleig et al., 2012) were processed by ectopically expressed RHBDL4 (Figure 2A). This suggests that RHBDL4 shows substrate specificity. As a follow-up, we tested two additional ERAD 
substrates resembling truncated type I membrane proteins, namely RI332, a deletion of ribophorin 1 (RPN1) (Tsao et al., 1992), and a loss-of-function splice variant of the $\beta$ secretase (BACE476 $\Delta$ ) (Tanahashi and Tabira, 2007). BACE476 $\Delta$ was cleaved by ectopically expressed RHBDL4 leading to a 50-kDa fragment that appears between the glycosylated full-length 54-kDa form of BACE476 $\Delta$ and the MG132-stabilized 45-kDa deglycosylated species (Figure 2B and S2A). Interestingly, ectopic expression of RHBDL4 diminished steady-state level of BACE476 $\Delta$ and completely depleted the MG132-sensitve deglycosylated full-length $45-k D a$ species. This suggests that upon overexpression, RHBDL4 interacts with its substrates before they approach $\mathrm{Hrd} 1$ and thereby outcompetes dislocation of unprocessed BACE476 $\Delta$. Consistent with a scenario of dislocating shorter, RHBDL4generated BACE fragments, an overexposed western blot reveals a 40-kDa BACE-peptide in response to MG132 treatment (Figure $2 \mathrm{~B}$ ). Although we previously observed that degradation kinetics in Hek293T cells were unaffected by RHBDL4 knockdown for RI332 (Fleig et al., 2012), processing of RI332 by an unknown ER protease had been observed before (Mueller et al., 2006). Consistent with this, co-expression of RI332 with RHBDL4 generated several RI332 fragments in the range of 25 to $35 \mathrm{kDa}$, whereas the SA mutant stabilized traces of the deglycosylated unprocessed species as previously observed (Figure 2C and S2B). Interestingly, the type I membrane protein RPN1, is a native RHBDL4 substrate (Knopf et al., submitted). In addition to canonical cleavage in the TM region, RPN1 is cleaved at the same position as the truncated RI332 ERAD substrate (Figure S2B). This indicates that substrate selection of soluble substrates occurs in a related manner to cleavage of membrane-anchored ectodomains. Taken together, these results show that in addition to unstable membrane-integral proteins (Fleig et al., 2012; Paschkowsky et al., 2018), RHBDL4 can cleave several, but not all, ERAD-L substrates.

\section{RHBDL4 cleaves MHC202 to facilitate its p97-dependent dislocation}

The observation that RHBDL4-SA functionally interacts with deglycosylated MHC202 (Figure $1 \mathrm{E}$ ) and RI332 species (Figure $2 \mathrm{C}$ and S2B) indicates that rhomboid-catalyzed cleavage and protein dislocation are somehow coupled. As the ER-integral metalloprotease ZMPSTE24 (Ste24 in yeast) has recently been shown to clear polypeptide chains that got stuck in the Sec61 translocon channel (Ast et al., 2016), we decided to confirm a luminal localization of $\mathrm{MHC} 202$ prior cleavage. Endo $\mathrm{H}$ analysis reveals that the RHBDL4-generated $\mathrm{N}$-terminal MHC202 fragment is glycosylated (Figure S1E), indicating that it is formed in the ER lumen. To discriminate between a putative translocation intermediate with the C-terminus facing the cytoplasm and a fully translocated protein, we generated a MHC202 construct harboring an additional glycosylation site (K197N) in the C-terminal region (Figure 3A). We reasoned that only fully translocated $\mathrm{MHC} 202$ would be glycosylated at this site. Western blot analysis of 
MHC202-K197N co-expressed with RHBDL4 showed an Endo H-sensitive C-terminal fragment (Figure 3A). Consistent results were obtained with an MHC202 mutant with a single C-terminal glycosylation site only (Figure S3A), corroborating that RHBDL4 cleaves fully translocated MHC202. To further prove that RHBDL4 deals with ERAD-L substrates, we confirmed ER localization of MHC202 under RHBDL4 knockdown conditions by immunofluorescence microscopy (Figure S3B). Consistent with accumulation in the ER, under RHBDL4 knockdown the MHC202 signal increased in cells with a reduced RHBDL4 signal, whereas only a weak signal was detected in control cells (Figure S3C). To reach the proteasome, RHBDL4-generated cleavage fragments have to be dislocated into the cytoplasm. For this purpose, RHBDL4 recruits p97 to the ER membrane via a conserved VBM motif at its cytoplasmic C-terminus (Fleig et al., 2012; Lim et al., 2016), but blocking this interplay leads to accumulation of RHBDL4-generated cleavage fragments in the ER fraction (Fleig et al., 2012). Consistent with this, the p97 inhibitor CB-5083 (Anderson et al., 2015) stabilized the 18-kDa N-terminal MHC202 fragment (Figure 3B). Addition of MG132 did not further increase recovery of the cleavage fragment, indicating that solely blocking p97 prevented clearance of RHBDL4 generated fragments by retaining them in the ER fraction and thereby impeding their access to the proteasome.

\section{Processing by RHBDL4 is determined by specific features and not general substrate ubiquitination}

Next, we asked what requirements a protein has to fulfill to be recognized by RHBDL4. Since FLAG-tagged full-length MHC class I heavy chain (MHC-FL, Figure 4A) was not cleaved by RHBDL4 (Figure 4B), we asked whether triggering substrate ubiquitination would make it prone for cleavage. We therefore took advantage of the fact that as part of an immune evasion strategy the human cytomegalovirus (HCMV) protein US11 targets MHC-FL towards ERAD E3 ubiquitin ligases (Wiertz et al., 1996). However, even though US11 prompted a higher turnover of MHC-FL (Figure S4), co-expression of RHBDL4 did not lead to any proteolytic processing (Figure 4B). This result shows that specific substrate features and not the general ubiquitination status and turnover rate determine recognition by RHBDL4. As previous work demonstrated that a TM degron is sufficient to induce RHBDL4-catalyzed cleavage (Fleig et al., 2012), we fused the luminal part of MHC to the TM domain and cytosolic tail of a known RHBDL4 substrate, the $\alpha$-chain of pre-T cell receptor (pT $\alpha$ ) (MHCpT $\alpha$, Figure 4A). Consistent with previous findings, the TM degron was sufficient for RHBDL4-recognition (Fleig et al., 2012) leading to efficient processing of the MHC-pT $\alpha$ fusion protein (Figure 4C). In addition to two prominent cleavage sites in the context of the TM region, we observed an 18-kDa fragment in the range of the MHC202 cleavage product. This result shows that the $\mathrm{C}$-terminal truncation of MHC202 is not strictly required for 
RHBDL4-catalyzed cleavage and different determinants can lead to the same productive interaction with RHBDL4.

RHBDL4 cleaves at defined site, but substrate selection is determined by additional features Processing of the membrane-anchored MHC-pT $\alpha$ in the same region as MHC202 supports the notion that RHBDL4 preferentially cleaves at certain amino acid residues. For bacterial rhomboid proteases a loose consensus sequence with small side chains at the scissile peptide bond has been shown to at least partially determine cleavage specificity (Strisovsky et al., 2009). Hence, we narrowed down the site of RHBDL4-catalyzed cleavage and then mutated small amino acids within this stretch to phenylalanines. For MHC202, cleavage by ectopically expressed RHBDL4 was abolished in a mutant with a deletion between amino acid 121 and amino acid 128 (Figure 4D). Within this stretch, four small amino acids are found in two pairs, namely glycine-121 (G121), cysteine-122 (C122), glycine-125 (G125) and serine-126 (S126). Only mutation of all four residues to phenylalanine (121FF,125FF) abolished cleavage completely, whereas mutating the second pair (125FF) partially reduced cleavage (Figure 4D). This result indicates that the major processing occurs at G125, but G121 provides an alternative cleavage site. Consistent with this, G125 is located at a surface exposed loop between two antiparallel $\beta$-sheets forming the hydrophobic interface of the $\alpha 1$ $\alpha 2$-domains to the juxtamembrane $\alpha 3$-domain in full length $\mathrm{MHC}$, which is deleted in MHC202 (Figure 1A and 4D). Interestingly, mutation of small residues in the MHC202 cleavage site region to proline, which for bacterial rhomboids has been shown to prevent processing of the nearby peptide bond (Strisovsky et al., 2009), increased RHBDL4catalyzed cleavage (Figure 4E). This was particular pronounced in the glycine-121-proline, serine-126-proline double mutant (PP), which on SDS-PAGE led to a major change in the MHC202 running behavior (Figure 4E), and leads in presence of RHBDL4 wild type to at least three additional cleavage products. Since proline is precited to break secondary structure elements, these results indicate that the cleavage site accessibility has a major impact on MHC202 processing. Overall, we provide evidence that RHBDL4 substrate selection is a multi-layer process with sequence-specific recognition of the scissile peptide bond contributing to specificity, but secondary structure and the overall protein stability playing a dominating role.

\section{The erlin ERAD complex interacts with RHBDL4 and MHC202}

As RHBDL4 did not primarily rely on the amino acid sequence, we wondered whether RHBDL4 assembles with other ERAD factors that contribute to substrate recruitment. A critical step in the analysis of membrane protein complexes is to combine efficient one-step affinity purification of proteins expressed at physiological levels. We therefore endogenously 
tagged RHBDL4 in Hek293T cells at its C-terminus with a single FLAG-tag using CRISPR/Cas12-mediated gene editing (Figure S5A-B) (Fueller et al., 2019). Hek293T cells expressing FLAG-tagged RHBDL4 were grown in medium supplemented with 'heavy' labeled amino acids whereas the parenteral Hek293T cells were cultured in normal medium. Subsequently, the same number of cells were mixed, RHBDL4-FLAG was isolated from Triton X-100 solubilized microsomes and co-purified interaction partners were identified by mass spectrometry (Figure 5A). The previously identified RHBDL4 co-factor p97 (Fleig et al., 2012) was 1.4-fold enriched, demonstrating the efficiency of this workflow. In order to identify core components of RHBDL4-dependent ERAD, we focused on proteins identified in all three replicates. Among the 20 proteins that showed enrichment in the RHBDL4-FLAG fraction greater than 1.4-fold were the chaperones BiP and calreticulin, two protein disulfide isomerases, PDI and Erp44, and both subunits of the regulatory glucosidase II (Table S1). Furthermore, a pair of two homologues membrane-integral ERAD factors, namely Erlin1 and Erlin2, were enriched by 1.5 -fold. We reasoned that the luminal quality control factors are likely co-purified with bound RHBDL4 substrates and focused on the erlins and asked whether they are part of a functional membrane protein complex. Consistent with a stable assembly, co-immunoprecipitation and western blotting confirmed co-purification of RHBDL4 with Erlin1 and Erlin2 (Figure 5B and S5C-E). Erlin1 and Erlin2 were previously demonstrated to form a MDa-ERAD complex that among other clients is involved in degradation of the IP(3) receptor (Huber et al., 2013; Inoue and Tsai, 2017; Pearce et al., 2009), suggesting that RHBDL4 functionally interacts with this ERAD sub-branch. The E3 ligase RNF170 that previously had been shown to interact with the erlin complex was also co-purified with ectopically expressed RHBDL4 (Figure S5F). Interestingly, Erlin2 showed stronger interaction with RHBDL4-GFP wild-type when compared to the catalytic inactive SA mutant (Figure 5C). This result suggests that Erlin2 is not trapped by the SA mutant as would be the case for a RHBDL4 substrate. Hence, we may speculate that erlins play a role in substrate recruitment - as a putative substrate adaptor may bind to RHBDL4 without a bound substrate to deliver proteins but potentially dissociates from a trapped, stalled rhomboid-substrate complex. Consistent with a functional interplay of RHBDL4 with the erlin complex, blue native polyacrylamide electrophoresis (BN-PAGE) of immunoisolated FLAGtagged RHBDL4 showed distinct complexes in the range of $250 \mathrm{kDa}$ to $1.2 \mathrm{MDa}$, with Erlin2 co-purifying and co-migrating with the largest assembly (Figure 5D and S5G). To test our hypothesis that erlins are substrate-adaptors, we asked whether Erlin1 and Erlin2 interact with MHC202. Indeed, immunoprecipitation of Erlin1-HA or Erlin2-HA pulled down FLAGtagged MHC202 but not the stable, secreted control protein Prl (Figure 5E). Consistent with a role of the erlin complex in the RHBDL4 pathway, knockout of Erlin2 should reduce turnover of MHC202 (Figure S5H and 5F). 


\section{RHBDL4 facilitates removal of aggregation-prone ERAD-L substrates}

In addition to canonical ERAD, Erlin2 was recently shown to act as a chaperone on an artificially designed, ER-targeted aggregation-prone protein called ER $\beta$ (Vincenz-Donnelly et al., 2018) (Figure S6A). As Erlin2 and RHBDL4 are part of one complex, we wondered whether RHBDL4 also interacts with and degrades ER $\beta$. Indeed, the catalytic inactive SA mutant of RHBDL4 traps ER $\beta$ resulting in co-immunoprecipitation of ER $\beta$ with RHBDL4-SA but not wild type (Figure 6A). This mirrors the behavior of RHBDL4 substrates like MHC202 (Figure 1E) or pT $\alpha$ (Fleig et al., 2012). Consistent with this, co-expression of RHBDL4 with $E R \beta$ increases generation of a C-terminal cleavage fragment (Figure 6B). This let us wonder whether RHBDL4 might be of general importance for turnover of aggregation-prone peptides. Interestingly, the disease-associated, aggregation-prone Aguadilla variant of the fibrinogen $\gamma$ chain harboring the arginine-375-tryptophane (R375W) substitution (Brennan et al., 2002; Kruse et al., 2006b) was cleaved four times more as the wild type $\gamma$-chain (Figures 6C and S6B). This suggests that, despite an almost unchanged amino acid sequence, the biophysical property of an aggregation-prone ERAD-L substrate targets $\gamma$-fibrinogen into the RHBDL4-dependent ERAD clipping pathway. Consistent with a role in facilitating clearance of aggregation-prone peptides, we observed that knockdown of RHBDL4, in addition to increasing the steady-state level of MHC202, also increases the level of MHC202 recovered in the Nonident P40 (NP40) insoluble fraction (Figure 6D). Taken together, these results reveal RHBDL4 as an important safeguard for ERAD-L. The molecular mechanism of how aggregation-prone protein conformations are recognized, and how RHBDL4-catalyzed clipping facilitates dislocation into the cytoplasm are important questions that remain to be solved in the future.

\section{Discussion}

Protein aggregation in cells is an abnormal condition that is associated with aging and human disorders ranging from diabetes to neurodegeneration (Labbadia and Morimoto, 2015; Reis-Rodrigues et al., 2012). While multiple safeguards are known to cope with cytoplasmic protein aggregates (Koga et al., 2011; Mogk et al., 2018), still little is known about pathways that clear aggregating proteins from the ER lumen. Our results show that the rhomboid protease RHBDL4 contributes to turnover of soluble, aggregation-prone ERAD-L substrates. While this substrate class commonly is degraded through the Hrd1-dependent ERAD dislocation route (Christianson and Ye, 2014; Ruggiano et al., 2014), aggregationprone conformations of the same substrates may be targeted to RHBDL4 for cleavage (Figure 7). We suggest that this rhomboid-catalyzed clipping mechanism may facilitate 
protein turnover by generating shorter fragments that are more easily dislocated into the cytoplasm for proteasomal degradation. Under conditions when RHBDL4-dependent ERAD is compromised, or the substrate-load exceeds its capacity, various ERAD-L substrates aggregate, highlighting the importance of this proteostasis mechanism.

\section{RHBDL4 binds erlins to target aggregation-prone ERAD-L substrates for degradation} Biochemical analysis suggests that rhomboid proteases do not need any invariant subunit and may act as single chain proteases (Lemberg et al., 2005; Urban and Wolfe, 2005) or dimers (Arutyunova et al., 2014). This is a striking difference to the aspartic intramembrane protease presenilin, which in order to become active has to assemble with three invariant subunits, Nicastrin, PEN2 and APH1, forming the $\gamma$-secretase complex (Edbauer et al., 2003; Kimberly et al., 2003; Takasugi et al., 2003). Here, we reveal by shotgun proteomics of genomically tagged RHBDL4 that the two substrate recruitment proteins Erlin1 and Erlin2 (Pearce et al., 2007; Pearce et al., 2009) are in a native complex with RHBDL4. A previous proximity proteomics approach did not reveal significant interaction of RHBDL4 to membrane integral components (Ikeda and Freeman, 2019). However, the study by Ikeda and Freeman used a BirA fused to RHBDL4's C-terminal tail that likely does not get in proximity to erlins that lack any prominent cytoplasmic portion (Pearce et al., 2009). In the light of our analysis of the native RHBDL4 interactome and a recent study on the mitochondrial rhomboid protease PARL (Wai et al., 2016), we may now speculate that also rhomboids form higherorder assemblies. The RHBDL4 complex shows a striking parallel to another intramembrane protease in the ER, the aspartic protease SPP (signal peptide peptidase), which exists in higher order complexes with ERAD components (Chen et al., 2014; Stagg et al., 2009). Our BN-PAGE analysis revealed several larger assemblies including a complex $>1 \mathrm{MDa}$ for RHBDL4 after solubilization with Triton X-100 containing endogenous Erlin2 and probably also the other subunits of the erlin complex. Previous work has shown interaction of erlins with ERAD substrates as diverse as the IP(3) receptor (Lu et al., 2011; Pearce et al., 2007; Pearce et al., 2009) and the artificially designed aggregation-prone luminal peptide ER $\beta$ (Vincenz-Donnelly et al., 2018). Intriguingly, the erlin complex forms an assembly similar to chaperonins, albeit without ATPase activity and was hypothesized to bind hydrophobic stretches that are a hallmark for aggregating proteins (Pearce et al., 2009). Combined with the RHBDL4-catalyzed clipping mechanism, this erlin recognition mechanism may help to lower protein aggregation in the ER lumen (Vincenz-Donnelly et al., 2018). While globular misfolded proteins are primarily targeted to the canonical Hrd1-Sel1 pathway (Christianson and Ye, 2014; Ruggiano et al., 2014), aggregation-prone peptide conformations may be recognized by erlins targeting certain ERAD-L substrates towards RHBDL4 for cleavage (Figure 7). Interestingly, erlins are members of the SPFH (stomatin, prohibitin, flotillin, $\mathrm{HfC} / \mathrm{K}$ ) 
family that also include Stomatin-like protein 2 (SLP2) (Browman et al., 2007; Browman et al., 2006). SLP2 in turn was shown to assemble in a 2-MDa complex with the mitochondrial rhomboid protease PARL and the $i$-AAA protease YME1L, where SLP2 is thought to regulate PARL-catalyzed intramembrane proteolysis (Wai et al., 2016). It remains to be seen whether erlins control RHBDL4 activity in a similar manner. Considering also that prohibitins, the closest relatives of erlins, form higher molecular weight complexes regulating $m$-AAA proteases (Steglich et al., 1999) this could likely be a mechanism shared by several proteases linked to proteostasis control. Overall, the emerging picture of RHBDL4 is that there are at least two different substrate recognition routes, one for membrane proteins and one for soluble ERAD-L substrates, both leading to clipping and subsequent degradation by the proteasome.

Recognition of soluble substrates by the membrane-integral rhomboid active site The crystal structures of the Escherichia coli rhomboid protease GIpG revealed the active site to be located several Ångstroms beneath the membrane surface, in the center of a six TM helix-bundle (Wang et al., 2006). A combination of structural and biochemical studies on bacterial rhomboids provided evidence for a lateral lipid-embedded substrate gate and a surface exposed active site opening, which is temporally shielded by a flexible loop structure (for review see (Ticha et al., 2018)). While helical, lipid-embedded substrate TM segments are thought to unfold into the active site via the membrane-embedded lateral gate (Cho et al., 2016), it is conceivable that for RHBDL4 the surface-active site opening allows flexible loops of ERAD-L substrates to enter the active site from the ER lumen. Accordantly, we and others observed rhomboid cleavage within ectodomains and loops of membrane proteins (Fleig et al., 2012; Knopf et al., submitted; Maegawa et al., 2007; Paschkowsky et al., 2018) and detergent solubilized rhomboids are known to cleave in vitro soluble model substrates (Arutyunova et al., 2018; Maegawa et al., 2005; Wang et al., 2006). Here, we now show cleavage of a soluble ERAD-L substrate in a physiological context. This unexpected wide substrate spectrum of an intramembrane protease poses the question of how specificity is achieved. In general, rhomboid proteases are thought to recognize their substrates by a combination of sampling TM domain stability and amino acid sequence (for review see (Langosch et al., 2015)). For efficient protease cleavage, the transition state of the enzymatic reaction is stabilized by tight binding of the amino acid residues surrounding the scissile peptide bond (Schechter and Berger, 1967). Sequence specific recognition of the scissile peptide bond has been observed for bacterial rhomboids (Strisovsky et al., 2009; Zoll et al., 2014), however, it is not entirely clear how substrates are selected in a biological context and whether other determinants such as substrate tails dominate specificity (Fleig et al., 2012; Lohi et al., 2004). For RHBDL4, we now showed that small side chains are required to 
promote cleavage. However, only mutation of all small amino acids in the cleavage site region prevented processing. Taken together, cleavage site recognition for RHBDL4 seems to be primarily determined by the secondary structure and only a very loose sequence motif determines the exact site of cleavage, whereby the overall discrimination between substrates and non-substrates may be regulated by ER quality control factors and ERAD substrate adaptors such as erlins (Figure 7).

\section{A role of RHBDL4 in oligomer removal}

Aggregates are higher molecular structures that are commonly no longer soluble in nonionic detergents (Valetti et al., 1991). Seen from this angle, the increase of NP40-insoluble MHC202 under RHBDL4 knockdown, first shows that MHC202 has the tendency to aggregate and second, it suggests that RHBDL4 is important for removal of aggregationprone proteins. A role of RHBDL4 in clipping aggregation-prone ERAD-L substrates is corroborated by our finding that the Aguadilla mutant of fibrinogen $\gamma$-chain, predestined to form aggregates (Kruse et al., 2006b), is cleaved three times more than the wild type protein. Likewise, we observed that the aggregation-prone model protein ER $\beta$ functionally interacts with RHBDL4. All together, these results suggest that RHBDL4, in cooperation with the erlin complex, cleaves and thereby induces the degradation of aggregation-prone ERAD-L substrates. In contrast, given the limited dimension of any putative ERAD dislocation channel, macroscopic protein aggregates can only be removed by autophagy- or vesiclebased, lysosomal degradation routes (Fregno et al., 2018; Fu and Sztul, 2009). In addition to controlling the integrity of the membrane proteome as previously described (Fleig et al., 2012), RHBDL4 serves as an important fail-safe mechanism for ER luminal protein homeostasis by lowering the concentration of aggregation-prone luminal ERAD-L substrates. Further insights into RHBDL4 complex composition and identification of additional endogenous substrates likely will unveil important cellular mechanisms. These insights will be indispensable to utilize the capacity of RHBDL4 in pre-aggregate removal for therapeutic application. 


\section{Materials and Methods}

\section{Plasmids and RNA interference}

Unless otherwise stated, all constructs were cloned into pcDNA3.1+ (Invitrogen). Human RHBDL4 (UniGene ID Hs.84236; IMAGE 40023929) was cloned with an N-terminal triple HA-tag as described (Fleig et al., 2012). For generating point mutants, a site-directed mutagenesis strategy was used. Constructs for C-terminal GFP-tagged human RHBDL4 were generated by subcloning the open reading frame into the monomeric variant of pEGFP$\mathrm{N} 1$ (Clontech). For affinity purification by immunoprecipitation and peptide elution, a Cterminal single FLAG-tagged mouse RHBDL4 was cloned. Plasmids encoding triple FLAGtagged RI332, secreted human prolactin and PrI-KDEL were described previously (Fleig et al., 2012). A truncated 202-amio acid long version of human MHC class I heavy chain A2 (UniProt ID 078126 with a C-terminal FLAG-tag was cloned into pCMV-S11 (Sandia BioTech). N-terminal triple FLAG-tagged versions of MHC-FL, MHC202 (comprising residues 25 to 202 of the MHC ORF), OS9 (UniGene ID Hs. 527861, IMAGE:2964645), NHK (gift from R. Kopito), BACE476 $\Delta$ (gift from M. Molinari), fibrinogen $\gamma$-chain wt and -R375W (gift from J. Brodsky) were generated by subcloning the respective open reading frames omitting their signal sequences into a pcDNA3-based expression vector containing a signal sequence fused to a triple FLAG-tag (Fleig et al., 2012). The glycosylation mutants MHC202-K197N and MHC202-N100Q-K197N were cloned with a C-terminal triple FLAG-tag followed by an S-tag. The MHC-pT $\alpha$ chimera was generated by overlap extension PCR, fusing residues 22304 of MHC-FL to the TM domain and C-terminus of $\mathrm{pT} \alpha$ (residues 147-281). For stable expression, FLAG-MHC202 was subcloned into pcDNA5/FRT/TO (Invitrogen). Myc-tagged HCMV strain AD169 US11 (UniProt ID P09727) was ordered after codon optimization as gBlock (IDT) and cloned into pcDNA3.1+. Constructs encoding FLAG-tagged RNF170, HAand FLAG-tagged human Erlin1 and Erlin2 (gift from R. Wojcikiewicz) (Pearce et al., 2007; Pearce et al., 2009) and Myc-tagged ER $\beta$ (gift from M. Hipp) (Vincenz-Donnelly et al., 2018), the ER marker RFP-KDEL (Altan-Bonnet et al., 2006) were described previously. For cleavage assays, ER $\beta$ was cloned with an N-terminal Myc and a C-terminal triple FLAG-tag into pcDNA3.1. For transient knockdown the small hairpin (shRNA)-expressing vectors pSUPER.neo (R4-1) (Fleig et al., 2012) and a pRS vector-based construct targeting 5'ATGAGGAGACAGCGGCTTCACAGATTCGA-3'(R4-2) (OriGene) were used. As nontargeting (nt) control pSUPER.neo targeting 5'-ACAGCUUGAGAGAGCUUUA-3' designed for knockdown of RHBDL4 in COS7 cells (but not human cells) was used. To generate RHBDL4 knockout Hek293T cells, TALEN expression vectors were obtained from www.talenlibrary.net (Kim et al., 2013) with the following sequences: left NGNGNNNGNINGNGNGHDNININGNINGNNNNHDNING-, right HDNGNGNGHDNGNINNNINGNGNGNINGNGHDHDNGNG-. 
For generating single guide (sgRNA) target sequences for Erlin2, the E-CRISPR tool (http://www.e-crisp.org) was used (Heigwer et al., 2014). The target sequence 5'CACCGGCTGTGCACAAGATAGAAGA-3' was then cloned in a Bbsl linearized px459.v2 vector containing puromycin selection. For the siRNA screen, an on Target Plus SMART pools custom library was used (Dharmacon).

\section{Cell lines and transfection}

Hek293T cells were cultured in DMEM (Invitrogen) complemented with 10\% fetal bovine serum at $37^{\circ} \mathrm{C}$ in $5 \% \mathrm{CO}_{2}$. Transient transfections were performed using $25 \mathrm{kDa}$ linear polyethylenimine (Polysciences) (Durocher et al., 2002) as had been described (Fleig et al., 2012). Typically, $500 \mathrm{ng}$ plasmid encoding substrate candidate and $100 \mathrm{ng}$ plasmid encoding RHBDL4 were used per well of a 6 -well plate. Total transfected DNA ( $2 \mu \mathrm{g} /$ well) was held constant by the addition of empty plasmid. If not otherwise stated, cells were harvested $48 \mathrm{~h}$ after transfection. For inhibition of the proteasome or p97, approx. $32 \mathrm{~h}$ post transfection either $2 \mu \mathrm{M}$ MG132 (Calbiochem) or $2.5 \mu \mathrm{M}$ CB-5083 (ApexBio) were added from a 10,000 x stock in dimethylsolfoxid (DMSO). As a vehicle control, the same amount of DMSO was used. Subsequently, cells were further incubated and harvested $16 \mathrm{~h}$ later. Cells were lysed in SDS-sample buffer (see below).

To prepare doxycycline inducible stably transfected cells pcDNA5/FRT/TO/FLAG-MHC202, FIp-In Hek293 T-REx cells were co-transfected with pOG44 (Invitrogen), followed by selection with hygromycin B $(125 \mu \mathrm{g} / \mathrm{ml})$. For generating RHBDL4 knockout cells, $2 \mu \mathrm{g}$ from each TALEN vector, or for generating Erlin2 knockout, $1 \mu \mathrm{g}$ of CRISPR/Cas9 vector were transfected into Hek293T. After 24 h, a single cell dilution was performed. Clones were analyzed by western blotting and sequencing of a PCR amplicon obtained from genomic DNA. Primers used for RHBDL4 were 5'-GCTGTTTCCCTGATATCTGG-3' and 5'GCAAAAACTAGTCCCTGCATG-3', leading to a 620-bp PCR product. Primers used for validation of Erlin2 knockout cells were: 5'- CTTGAGCAACGGCTGTATCC-3' and 5'AATCACCACCCATGGCATCAT-3' leading to a 610 bp amplicon. Generation of chromosomally tagged RHBDL4-FLAG Hek293T cells with a single FLAG before the stop codon in the last exon by using CRISPR/Cas12 mediated gene editing has been described before (Fueller et al., 2019). Primers used for validation were: 5'-

TTATGGAGCACGATGGAAGGAA-3' and 5'-GAGATGGGAGCGTGGAAACT-3', leading to a 634 bp amplicon.

\section{Antibodies}


The following antibodies were used: mouse monoclonal anti-FLAG (M2, Sigma), rat monoclonal anti-HA (Roche), mouse anti-myc (New England Biolabs), rabbit polyclonal antiGFP (gift from Oliver Gruss) and mouse monoclonal anti GFP (Roche), mouse monoclonal anti- $\beta$ actin (Sigma), mouse monoclonal anti-Derlin1 (Sigma), rabbit polyclonal anti-p97 (gift from Bernd Dobberstein), rabbit polyclonal anti-H2B (Abcam), mouse monoclonal antiCLIMP63 (Enzo Life Sciences) rabbit polyclonal anti-Erlin2 (Sigma), rabbit polyclonal antiRHBDL4 (Sigma) and rabbit polyclonal anti-RHBDL4 (Fleig et al., 2012).

\section{Microscopy}

For immunofluorescence analysis, cells were either chemically fixed in PBS containing 4\% paraformaldehyde for 30 min followed by permeabilization in PBS containing $0.5 \%$ Triton X100 for $10 \mathrm{~min}$ (Figure S3B) or fixed in methanol at $-20^{\circ} \mathrm{C}$ for 5 minutes (Figure S3C). Subsequently, cells were washed with PBS, blocked with $20 \%$ fetal calf serum in PBS and probed with affinity-purified anti RHBDL4 antibody (1:50; see above) and anti-FLAG antibody (1:1000). After staining with fluorescently labeled secondary antibody (Santa Cruz Biotechnology), slides were analyzed using a TCS SP5 confocal microscope (Leica).

\section{NP40 solubility assay}

To test the influence of RHBDL4 on solubility of proteins, 300 ng FLAG-MHC202 expressing vector was transfected with $1000 \mathrm{ng}$ shRNA and $700 \mathrm{ng}$ empty vector. After $48 \mathrm{~h}$ of transfection, cells were pelleted and solubilized in NP40 lysis buffer (50 mM Tris-Cl, pH 7.4, $150 \mathrm{mM} \mathrm{NaCl}, 2 \mathrm{mM} \mathrm{MgCl} 2,1 \%$ Nonidet P-40) supplemented with 1XPI. After $5 \mathrm{~min}$ centrifugation at full speed at $4^{\circ} \mathrm{C}$, supernatant corresponding to the soluble fraction was transferred into new tube containing $4 \mathrm{x}$ sample buffer (see below). Pellet was dissolved in $1 \mathrm{x}$ sample buffer and corresponds to insoluble fraction.

\section{Cycloheximide chase}

Cycloheximide $(100 \mu \mathrm{g} / \mathrm{ml})$ chase was conducted $24 \mathrm{~h}$ after transfection of Hek293T cells and cell extracts were subjected to western blot analysis as described below.

\section{Immunoprecipitation and proteomics}

If not indicated differently, all steps were performed at $4^{\circ} \mathrm{C}$. For substrate trapping, RHBDL4GFP expressing Hek293T cells were solubilized with $1 \%$ Triton X-100 in IP buffer (50 mM HEPES-KOH, pH 7.4, $150 \mathrm{mM} \mathrm{NaCl}, 2$ mM MgOAc $2,10 \%$ glycerol, 1 mM EGTA), containing $1 \mathrm{XPI}$ and $10 \mu \mathrm{g} / \mathrm{ml}$ PMSF. Cell lysates were cleared by centrifugation at 10,000 $\mathrm{g}$ for $10 \mathrm{~min}$, following pre-clearing for $1 \mathrm{~h}$ with BSA-coupled sepharose beads or protein A/G beads. AntiGFP immunoprecipitation was performed using a monoclonal GFP-specific antibody in 
combination with protein $\mathrm{G}$ beads (Figure 1E) or GFP-specific single chain antibody fragment (Rothbauer et al., 2008) coupled to NHS-activated sepharose beads (Figure 5C) as described (Fleig et al., 2012). For immunoprecipitation of HA-tagged proteins, anti-HA antibody-coupled agarose beads (Sigma) were used. For immunoprecipitation of endogenous RHBDL4, the primary antibody was added together with protein A beads for overnight incubation. Immunoprecipitates were washed three times in IP buffer containing $0.1 \%$ Triton $\mathrm{X}-100$ and then resuspended in SDS sample buffer followed by SDS-PAGE and western blotting (see below).

For isolation of endogenous RHBDL4 interaction partners by shotgun proteomics, Hek293TRHBDL4-FLAG cells were grown for at least six doublings in medium supplemented with heavy amino acids $\left({ }^{13} \mathrm{C}_{6}{ }^{15} \mathrm{~N}_{4}\right.$-L-Arg and ${ }^{13} \mathrm{C}_{6}{ }^{15} \mathrm{~N}_{2}$-L-Lys, from Silantes), whereas the parenteral Hek293T cells cultured in light medium were used as control. The third replicate was performed with a label swap to minimize the experimental error. After harvesting, equal number of cells from both cultures were mixed and pooled microsome fraction was isolated by hypotonic swelling and centrifugation as previously described (Chen et al., 2014). For immunoprecipitation of RHBDL4-FLAG, microsomes were solubilized with $1 \%$ Triton X-100 in IP buffer, containing $1 \mathrm{xPI}$ and $10 \mu \mathrm{g} / \mathrm{ml}$ PMSF. Cell lysates were cleared by centrifugation at $20,000 \mathrm{~g}$ for $10 \mathrm{~min}$. Pre-clearing with protein A beads and anti-FLAG immunoprecipitation was performed as described above. The immunocomplexes were eluted in SDS sample buffer and resolved by SDS-PAGE. The lane was subdivided into three pieces and an in-gel trypsin digest was performed. First proteins were reduced with DTT, alkylated with iodacedamide and then digested with trypsin. Following digestion, peptides were extracted with $50 \%$ acetonitrile/0.1\% TFA and concentrated in a SpeedVac vacuum centrifuge. The sample was analyzed by a UPLC system (nanoAcquity) coupled to an ESI LTQ Oribitrap mass spectrometer (Thermo). The uninterpreted MS/MS spectra were searched against the SwissProt-human database using MaxQuant software. The algorithm was set to use trypsin as enzyme, allowing at maximum for two missed cleavage site and assuming carbamidomethyl as a fixed modification of cysteine, and oxidized methionine and deamidation of asparagines and glutamine as variable modifications. Mass tolerance was set to $4.5 \mathrm{ppm}$ and 0.2 Da for MS and MS/MS, respectively. In MaxQuant the 'requantify' and 'match between runs' option was utilized, the target decoy method was used to determine $1 \%$ false discovery rate. All analysis was performed on the "protein groups" file using Perseus software version 1.6.5.0 (Tyanova et al., 2016) and Microsoft Excel. Label-free intensities were used to calculate the heavy over light ratios which were averaged over all three biological replicates. $P$ values of $\log _{2}$ transformed data were determined by one-sample 
t-test. The cutoff for a protein to be called significantly enriched was set to fold change $>1.4$ and $p$-value $<0.05$.

\section{Blue native PAGE}

If not indicated differently, all steps were performed at $4^{\circ} \mathrm{C}$. Hek293T cells ectopically expressing RHBDL4-FLAG or expressing chromosomally FLAG-tagged RHBDL4 were lysed with 1\% Triton X-100 in BN buffer (50 mM HEPES-KOH, $\mathrm{pH} 7.4,150 \mathrm{mM} \mathrm{NaCl}, 2 \mathrm{mM}$ $\mathrm{MgOAc}_{2}, 10 \%$ glycerol, 1 mM EGTA) supplemented with EDTA-free complete protease inhibitor cocktail (1xPI, Roche) and $10 \mu \mathrm{g} / \mathrm{ml}$ PMSF. After removal of cell debris, $10 \mu \mathrm{l}$ antiFLAG antibody conjugated agarose beads (M2, Sigma) were added. After a $3 \mathrm{~h}$ incubation, beads were washed twice with BN buffer containing $0.2 \%$ Triton X-100 and subsequently eluted with $0.5 \mu \mathrm{g} / \mu \mathrm{l}$ FLAG peptide for $30 \mathrm{~min}$. A $1 / 40$ volume of $B N$ sample buffer $(500 \mathrm{mM}$ 6-aminohexanoic acid, $100 \mathrm{mM}$ bis Tris pH 7.0, 5\% Coomassie G250) was added before subjection onto NativePAGE Novex Bis-Tris 3-12\% gradient gels (Thermo). Gels were run for $1 \mathrm{~h}$ at $150 \mathrm{~V}$, buffer changed according to the manufacturers description and then continued at $230 \mathrm{~V}$ for $45 \mathrm{~min}$. Afterwards, gels were incubated for $15 \mathrm{~min}$ in blotting buffer, then transferred at $85 \mathrm{~mA}$ for 70 min onto PVDF membrane using a tank-blotting system. The PVDF membrane was incubated in fixation solution (40\% methanol, $10 \%$ acetic acid), blocked in 5\% milk TBS-Tween (10 mM Tris-Cl pH 7.4, $150 \mathrm{mM} \mathrm{NaCl}, 0.1 \%$ Tween 20), and analyzed using enhanced chemiluminescence (see below).

\section{Western blotting}

Transfected cells and immunoisolated proteins were solubilized in Tris-glycine SDS-PAGE sample buffer (50 mM Tris-Cl pH 6.8, 10 mM EDTA, 5\% glycerol, 2\% SDS, 0.01\% bromphenol blue, $5 \% \beta$-mercaptoethanol). All samples were incubated for $15 \mathrm{~min}$ at $65^{\circ} \mathrm{C}$. For deglycosylation, solubilized proteins were treated with Endo $\mathrm{H}$ and PNGase F (New England Biolabs) according to the manufacturer's protocol. Denaturated and fully-reduced proteins were resolved on Tris-glycine SDS-PAGE followed by western blot analysis onto PVDF membrane (Immobilon-P, $0.45 \mu \mathrm{M}$ pore size, Merck Millipore) using enhanced chemiluminescence to detect bound antibodies (Pierce). For the analysis of ER $\beta$-derived cleavage fragments $(<10 \mathrm{kDa})$, post-nuclear supernatants from Triton X-100 solubilized cells were mixed with Tris-bicine-urea SDS-sample buffer (360 mM BisTris, $160 \mathrm{mM}$ bicine, 1\% SDS, $50 \mathrm{mM}$ dithiothreitol, 15\% sucrose, 0.01\% bromphenol blue, and 0.004\% Serva blue), heated at $65^{\circ} \mathrm{C}$. Peptides were separated to Tris/Bicine-urea PAGE (15\% T, 5\% C, 8 M urea) (Wiltfang et al., 1997), transfer onto PVDF membrane with $0.2 \mu \mathrm{m}$ pore size and analyzed by western blotting. For detection, the LAS-4000 system (Fuji) was used. Data shown are 
representative of three independent experiments. For quantification, we used the ImageJ software.

\section{Acknowledgments}

We gratefully acknowledge the contribution of Lina Wunderle to the beginning of this project. We thank Richard Wojcikiewicz, Ron Kopito, Maurizio Molinari, Jeff Brodsky and Mark Hipp for reagents. We thank Matthias Feige and Sebastian Schuck for critical reading of the manuscript. Mass spectrometry was performed at the ZMBH Core facility for mass spectrometry and proteomics. This study was supported by the Deutsche Forschungsgemeinschaft (DFG) Collaborative Research Center SFB1036-TP12 (to MKL), a fellowship by the Boehringer Ingelheim Fonds (to JDK) and an intramural research program of the National Institute of Diabetes, Digestive \& Kidney Diseases in the National Institutes of Health (to YY).

\section{Author contribution}

NK and JB designed and performed most experiments and wrote the manuscript. JDK carried out experiments. JGL performed and validated the siRNA screen. NL initiated the interactome analysis and validated interaction partners. $Y Y$ helped designing the project. MKL guided the project and wrote the manuscript.

\section{References}

Abe, F., Van Prooyen, N., Ladasky, J.J., and Edidin, M. (2009). Interaction of Bap31 and $\mathrm{MHC}$ class I molecules and their traffic out of the endoplasmic reticulum. Journal of immunology (Baltimore, Md. : 1950) 182, 4776-4783.

Altan-Bonnet, N., Sougrat, R., Liu, W., Snapp, E.L., Ward, T., and Lippincott-Schwartz, J. (2006). Golgi inheritance in mammalian cells is mediated through endoplasmic reticulum export activities. Mol Biol Cell 17, 990-1005.

Anderson, D.J., Le Moigne, R., Djakovic, S., Kumar, B., Rice, J., Wong, S., Wang, J., Yao, B., Valle, E., Kiss von Soly, S., et al. (2015). Targeting the AAA ATPase p97 as an Approach to Treat Cancer through Disruption of Protein Homeostasis. Cancer cell 28, 653-665.

Arutyunova, E., Jiang, Z., Yang, J., Kulepa, A.N., Young, H.S., Verhelst, S., O'Donoghue, A.J., and Lemieux, M.J. (2018). An internally quenched peptide as a new model substrate for rhomboid intramembrane proteases. Biological chemistry.

Arutyunova, E., Panwar, P., Skiba, P.M., Gale, N., Mak, M.W., and Lemieux, M.J. (2014). Allosteric regulation of rhomboid intramembrane proteolysis. EMBO J 33, 1869-1881. 
Ast, T., Michaelis, S., and Schuldiner, M. (2016). The Protease Ste24 Clears Clogged Translocons. Cell 164, 103-114.

Avci, D., and Lemberg, M.K. (2015). Clipping or Extracting: Two Ways to Membrane Protein Degradation. Trends Cell Biol 25, 611-622.

Balchin, D., Hayer-Hartl, M., and Hartl, F.U. (2016). In vivo aspects of protein folding and quality control. Science 353, aac4354.

Baldridge, R.D., and Rapoport, T.A. (2016). Autoubiquitination of the Hrd1 Ligase Triggers Protein Retrotranslocation in ERAD. Cell 166, 394-407.

Bernasconi, R., Galli, C., Calanca, V., Nakajima, T., and Molinari, M. (2010). Stringent requirement for HRD1, SEL1L, and OS-9/XTP3-B for disposal of ERAD-LS substrates. J Cell Biol 188, 223-235.

Bonifacino, J.S., Suzuki, C.K., and Klausner, R.D. (1990). A peptide sequence confers retention and rapid degradation in the endoplasmic reticulum. Science 247, 79-82.

Brennan, S.O., Maghzal, G., Shneider, B.L., Gordon, R., Magid, M.S., and George, P.M. (2002). Novel fibrinogen gamma375 Arg-->Trp mutation (fibrinogen aguadilla) causes hepatic endoplasmic reticulum storage and hypofibrinogenemia. Hepatology (Baltimore, Md.) $36,652-658$.

Breydo, L., and Uversky, V.N. (2015). Structural, morphological, and functional diversity of amyloid oligomers. FEBS Lett 589, 2640-2648.

Browman, D.T., Hoegg, M.B., and Robbins, S.M. (2007). The SPFH domain-containing proteins: more than lipid raft markers. Trends Cell Biol 17, 394-402.

Browman, D.T., Resek, M.E., Zajchowski, L.D., and Robbins, S.M. (2006). Erlin-1 and erlin-2 are novel members of the prohibitin family of proteins that define lipid-raft-like domains of the ER. J Cell Sci 119, 3149-3160.

Bulek, A.M., Cole, D.K., Skowera, A., Dolton, G., Gras, S., Madura, F., Fuller, A., Miles, J.J., Gostick, E., Price, D.A., et al. (2012). Structural basis for the killing of human beta cells by CD8(+) T cells in type 1 diabetes. Nat Immunol 13, 283-289.

Carvalho, P., Goder, V., and Rapoport, T.A. (2006). Distinct ubiquitin-ligase complexes define convergent pathways for the degradation of ER proteins. Cell 126, 361-373.

Chen, C., Malchus, N.S., Hehn, B., Stelzer, W., Avci, D., Langosch, D., and Lemberg, M.K. (2014). Signal peptide peptidase functions in ERAD to cleave the unfolded protein response regulator XBP1u. EMBO J 33, 2492-2506.

Chiti, F., and Dobson, C.M. (2017). Protein Misfolding, Amyloid Formation, and Human Disease: A Summary of Progress Over the Last Decade. Annu Rev Biochem 86, 27-68.

Cho, S., Dickey, S.W., and Urban, S. (2016). Crystal Structures and Inhibition Kinetics Reveal a Two-Stage Catalytic Mechanism with Drug Design Implications for Rhomboid Proteolysis. Mol Cell 61, 329-340.

Christianson, J.C., Olzmann, J.A., Shaler, T.A., Sowa, M.E., Bennett, E.J., Richter, C.M., Tyler, R.E., Greenblatt, E.J., Wade Harper, J., and Kopito, R.R. (2011). Defining human ERAD networks through an integrative mapping strategy. Nat Cell Biol 14, 93105. 
Christianson, J.C., and Ye, Y. (2014). Cleaning up in the endoplasmic reticulum: ubiquitin in charge. Nat Struct Mol Biol 21, 325-335.

Denic, V., Quan, E.M., and Weissman, J.S. (2006). A luminal surveillance complex that selects misfolded glycoproteins for ER-associated degradation. Cell 126, 349-359.

Durocher, Y., Perret, S., and Kamen, A. (2002). High-level and high-throughput recombinant protein production by transient transfection of suspension-growing human 293-EBNA1 cells. Nucleic Acids Res 30, E9.

Edbauer, D., Winkler, E., Regula, J.T., Pesold, B., Steiner, H., and Haass, C. (2003). Reconstitution of g-secretase activity. Nat. Cell Biol. 5, 486-488.

Fleig, L., Bergbold, N., Sahasrabudhe, P., Geiger, B., Kaltak, L., and Lemberg, M.K. (2012). Ubiquitin-Dependent Intramembrane Rhomboid Protease Promotes ERAD of Membrane Proteins. Mol Cell 47, 558-569.

Fregno, I., Fasana, E., Bergmann, T.J., Raimondi, A., Loi, M., Solda, T., Galli, C., D'Antuono, R., Morone, D., Danieli, A., et al. (2018). ER-to-lysosome-associated degradation of proteasome-resistant ATZ polymers occurs via receptor-mediated vesicular transport. Embo j.

Fu, L., and Sztul, E. (2009). ER-associated complexes (ERACs) containing aggregated cystic fibrosis transmembrane conductance regulator (CFTR) are degraded by autophagy. European journal of cell biology 88, 215-226.

Fueller, J., Meurer, M., Herbst, K., Gubicza, K., Kurtulmus B., Knopf, J.D., Kirrmaier, D., Buchmuller, B., Pereira, G., Lemberg, M.K., et al. (2019). CRISPR/Cas12a-assisted PCR tagging of mammalian genes (https://doi.org/10.1101/473876, bioRxiv).

Greenblatt, E.J., Olzmann, J.A., and Kopito, R.R. (2011). Derlin-1 is a rhomboid pseudoprotease required for the dislocation of mutant alpha-1 antitrypsin from the endoplasmic reticulum. Nat Struct Mol Biol 18, 1147-1152.

Grumati, P., Dikic, I., and Stolz, A. (2018). ER-phagy at a glance. J Cell Sci 131.

Heigwer, F., Kerr, G., and Boutros, M. (2014). E-CRISP: fast CRISPR target site identification. Nature methods 11, 122-123.

Hirsch, C., Blom, D., and Ploegh, H.L. (2003). A role for N-glycanase in the cytosolic turnover of glycoproteins. EMBO J 22, 1036-1046.

Hosokawa, N., Tremblay, L.O., You, Z., Herscovics, A., Wada, I., and Nagata, K. (2003). Enhancement of endoplasmic reticulum (ER) degradation of misfolded Null Hong Kong alpha1-antitrypsin by human ER mannosidase I. J Biol Chem 278, 26287-26294.

Huber, M.D., Vesely, P.W., Datta, K., and Gerace, L. (2013). Erlins restrict SREBP activation in the ER and regulate cellular cholesterol homeostasis. J Cell Biol 203, 427-436.

Ikeda, K.N., and Freeman, M. (2019). Spatial proteomics reveal that the protein phosphatase PTP1B interacts with and may modify tyrosine phosphorylation of the rhomboid protease RHBDL4. J Biol Chem.

Inoue, T., and Tsai, B. (2017). Regulated Erlin-dependent release of the B12 transmembrane J-protein promotes ER membrane penetration of a non-enveloped virus. PLoS Pathog 13, e1006439. 
Juszkiewicz, S., and Hegde, R.S. (2018). Quality Control of Orphaned Proteins. Mol Cell 71, 443-457.

Kim, Y., Kweon, J., Kim, A., Chon, J.K., Yoo, J.Y., Kim, H.J., Kim, S., Lee, C., Jeong, E., Chung, E., et al. (2013). A library of TAL effector nucleases spanning the human genome. Nat Biotechnol 31, 251-258.

Kimberly, W.T., LaVoie, M.J., Ostaszewski, B.L., Ye, W., Wolfe, M.S., and Selkoe, D.J. (2003). Gamma-secretase is a membrane protein complex comprised of presenilin, nicastrin, Aph-1, and Pen-2. Proceedings of the National Academy of Sciences of the United States of America 100, 6382-6387.

Knopf, J.D., Landscheidt, N., Pegg, C.L., Schulz, B.L., Kühnle, N., Chao, C.W., Huck, S., and Lemberg, M.K. (submitted). Intramembrane protease RHBDL4 induces ER-associated degradation of the oligosaccharyltransferase complex

Koga, H., Kaushik, S., and Cuervo, A.M. (2011). Protein homeostasis and aging: The importance of exquisite quality control. Ageing research reviews 10, 205-215.

Kruse, K.B., Brodsky, J.L., and McCracken, A.A. (2006a). Characterization of an ERAD gene as VPS30/ATG6 reveals two alternative and functionally distinct protein quality control pathways: one for soluble $Z$ variant of human alpha-1 proteinase inhibitor (A1PiZ) and another for aggregates of A1PiZ. Mol Biol Cell 17, 203-212.

Kruse, K.B., Dear, A., Kaltenbrun, E.R., Crum, B.E., George, P.M., Brennan, S.O., and McCracken, A.A. (2006b). Mutant fibrinogen cleared from the endoplasmic reticulum via endoplasmic reticulum-associated protein degradation and autophagy: an explanation for liver disease. The American journal of pathology 168, 1299-1308; quiz 1404-1295.

Kühnle, N., Dederer, V., and Lemberg, M.K. (2019). Intramembrane proteolysis at a glance: from signalling to protein degradation. . J. Cell Sci. , in press.

Labbadia, J., and Morimoto, R.I. (2015). The biology of proteostasis in aging and disease. Annu Rev Biochem 84, 435-464.

Langosch, D., Scharnagl, C., Steiner, H., and Lemberg, M.K. (2015). Understanding intramembrane proteolysis: from protein dynamics to reaction kinetics Trends Biochem Sci $40,318-327$.

Lemberg, M.K., Menendez, J., Misik, A., Garcia, M., Koth, C.M., and Freeman, M. (2005). Mechanism of intramembrane proteolysis investigated with purified rhomboid proteases. EMBO J. 24, 464-472.

Lim, J.J., Lee, Y., Ly, T.T., Kang, J.Y., Lee, J.G., An, J.Y., Youn, H.S., Park, K.R., Kim, T.G., Yang, J.K., et al. (2016). Structural insights into the interaction of p97 $\mathrm{N}$-terminus domain and VBM in rhomboid protease, RHBDL4. Biochem J 473, 2863-2880.

Lohi, O., Urban, S., and Freeman, M. (2004). Diverse substrate recognition mechanisms for rhomboids; thrombomodulin is cleaved by Mammalian rhomboids. Curr. Biol. 14, 236-241.

Lu, J.P., Wang, Y., Sliter, D.A., Pearce, M.M., and Wojcikiewicz, R.J. (2011). RNF170 protein, an endoplasmic reticulum membrane ubiquitin ligase, mediates inositol 1,4,5trisphosphate receptor ubiquitination and degradation. J Biol Chem 286, 24426-24433.

Maegawa, S., Ito, K., and Akiyama, Y. (2005). Proteolytic action of GlpG, a rhomboid protease in the Escherichia coli cytoplasmic membrane. Biochemistry 44, 13543-13552. 
Maegawa, S., Koide, K., Ito, K., and Akiyama, Y. (2007). The intramembrane active site of $\mathrm{GlpG}$, an E. coli rhomboid protease, is accessible to water and hydrolyses an extramembrane peptide bond of substrates. Mol. Microbiol. 64, 435-447.

McCaffrey, K., and Braakman, I. (2016). Protein quality control at the endoplasmic reticulum. Essays in biochemistry 60, 227-235.

Mogk, A., Bukau, B., and Kampinga, H.H. (2018). Cellular Handling of Protein Aggregates by Disaggregation Machines. Mol Cell 69, 214-226.

Mueller, B., Lilley, B.N., and Ploegh, H.L. (2006). SEL1L, the homologue of yeast Hrd3p, is involved in protein dislocation from the mammalian ER. J Cell Biol 175, 261-270.

Paschkowsky, S., Recinto, S.J., Young, J.C., Bondar, A.N., and Munter, L.M. (2018). Membrane cholesterol as regulator of human rhomboid protease RHBDL4. J Biol Chem.

Pearce, M.M., Wang, Y., Kelley, G.G., and Wojcikiewicz, R.J. (2007). SPFH2 mediates the endoplasmic reticulum-associated degradation of inositol 1,4,5-trisphosphate receptors and other substrates in mammalian cells. J Biol Chem 282, 20104-20115.

Pearce, M.M., Wormer, D.B., Wilkens, S., and Wojcikiewicz, R.J. (2009). An endoplasmic reticulum (ER) membrane complex composed of SPFH1 and SPFH2 mediates the ERassociated degradation of inositol 1,4,5-trisphosphate receptors. J Biol Chem 284, 1043310445.

Reis-Rodrigues, P., Czerwieniec, G., Peters, T.W., Evani, U.S., Alavez, S., Gaman, E.A., Vantipalli, M., Mooney, S.D., Gibson, B.W., Lithgow, G.J., et al. (2012). Proteomic analysis of age-dependent changes in protein solubility identifies genes that modulate lifespan. Aging cell $11,120-127$.

Rothbauer, U., Zolghadr, K., Muyldermans, S., Schepers, A., Cardoso, M.C., and Leonhardt, H. (2008). A versatile nanotrap for biochemical and functional studies with fluorescent fusion proteins. Mol Cell Proteomics 7, 282-289.

Ruggiano, A., Foresti, O., and Carvalho, P. (2014). Quality control: ER-associated degradation: protein quality control and beyond. J Cell Biol 204, 869-879.

Schechter, I., and Berger, A. (1967). On the size of the active site in proteases. I. Papain. Biochem Biophys Res Commun 27, 157-162.

Schoebel, S., Mi, W., Stein, A., Ovchinnikov, S., Pavlovicz, R., DiMaio, F., Baker, D., Chambers, M.G., Su, H., Li, D., et al. (2017). Cryo-EM structure of the protein-conducting ERAD channel Hrd1 in complex with Hrd3. Nature 548, 352-355.

Stagg, H.R., Thomas, M., van den Boomen, D., Wiertz, E.J., Drabkin, H.A., Gemmill, R.M., and Lehner, P.J. (2009). The TRC8 E3 ligase ubiquitinates MHC class I molecules before dislocation from the ER. J Cell Biol 186, 685-692.

Steglich, G., Neupert, W., and Langer, T. (1999). Prohibitins regulate membrane protein degradation by the m-AAA protease in mitochondria. Mol Cell Biol 19, 3435-3442.

Strisovsky, K., Sharpe, H.J., and Freeman, M. (2009). Sequence-specific intramembrane proteolysis: identification of a recognition motif in rhomboid substrates. Mol Cell 36, 10481059. 
Takasugi, N., Tomita, T., Hayashi, I., Tsuruoka, M., Niimura, M., Takahashi, Y., Thinakaran, G., and Iwatsubo, T. (2003). The role of presenilin cofactors in the gamma-secretase complex. Nature 422, 438-441.

Tanahashi, H., and Tabira, T. (2007). A novel beta-site amyloid precursor protein cleaving enzyme (BACE) isoform regulated by nonsense-mediated mRNA decay and proteasomedependent degradation. Neurosci Lett 428, 103-108.

Ticha, A., Collis, B., and Strisovsky, K. (2018). The Rhomboid Superfamily: Structural Mechanisms and Chemical Biology Opportunities. Trends Biochem Sci 43, 726-739.

Tsao, Y.S., Ivessa, N.E., Adesnik, M., Sabatini, D.D., and Kreibich, G. (1992). Carboxy terminally truncated forms of ribophorin I are degraded in pre-Golgi compartments by a calcium-dependent process. J Cell Biol 116, 57-67.

Tyanova, S., Temu, T., Sinitcyn, P., Carlson, A., Hein, M.Y., Geiger, T., Mann, M., and Cox, J. (2016). The Perseus computational platform for comprehensive analysis of (prote)omics data. Nature methods 13, 731-740.

Urban, S., and Wolfe, M.S. (2005). Reconstitution of intramembrane proteolysis in vitro reveals that pure rhomboid is sufficient for catalysis and specificity. Proc. Natl. Acad. Sci. USA 102, 1883-1888.

Valetti, C., Grossi, C.E., Milstein, C., and Sitia, R. (1991). Russell bodies: a general response of secretory cells to synthesis of a mutant immunoglobulin which can neither exit from, nor be degraded in, the endoplasmic reticulum. J Cell Biol 115, 983-994.

van der Goot, A.T., Pearce, M.M.P., Leto, D.E., Shaler, T.A., and Kopito, R.R. (2018). Redundant and Antagonistic Roles of XTP3B and OS9 in Decoding Glycan and Non-glycan Degrons in ER-Associated Degradation. Mol Cell 70, 516-530 e516.

Vashist, S., and Ng, D.T. (2004). Misfolded proteins are sorted by a sequential checkpoint mechanism of ER quality control. J Cell Biol 165, 41-52.

Vincenz-Donnelly, L., Holthusen, H., Korner, R., Hansen, E.C., Presto, J., Johansson, J., Sawarkar, R., Hartl, F.U., and Hipp, M.S. (2018). High capacity of the endoplasmic reticulum to prevent secretion and aggregation of amyloidogenic proteins. Embo j 37, 337-350.

Wai, T., Saita, S., Nolte, H., Muller, S., Konig, T., Richter-Dennerlein, R., Sprenger, H.G., Madrenas, J., Muhlmeister, M., Brandt, U., et al. (2016). The membrane scaffold SLP2 anchors a proteolytic hub in mitochondria containing PARL and the i-AAA protease YME1L. EMBO Rep 17, 1844-1856.

Wang, Y., Zhang, Y., and Ha, Y. (2006). Crystal structure of a rhomboid family intramembrane protease. Nature 444, 179-180.

Wiertz, E.J., Jones, T.R., Sun, L., Bogyo, M., Geuze, H.J., and Ploegh, H.L. (1996). The human cytomegalovirus US11 gene product dislocates MHC class I heavy chains from the endoplasmic reticulum to the cytosol. Cell 84, 769-779.

Wiltfang, J., Smirnov, A., Schnierstein, B., Kelemen, G., Matthies, U., Klafki, H.W., Staufenbiel, M., Huther, G., Ruther, E., and Kornhuber, J. (1997). Improved electrophoretic separation and immunoblotting of b-amyloid $(\mathrm{Ab})$ peptides 1-40, 1-42, and 1-43. Electrophoresis 18, 527-532.

Wu, X., and Rapoport, T.A. (2018). Mechanistic insights into ER-associated protein degradation. Curr Opin Cell Biol 53, 22-28. 
Wunderle, L., Knopf, J.D., Kuhnle, N., Morle, A., Hehn, B., Adrain, C., Strisovsky, K., Freeman, M., and Lemberg, M.K. (2016). Rhomboid intramembrane protease RHBDL4 triggers ER-export and non-canonical secretion of membrane-anchored TGFalpha. Scientific reports $6,27342$.

Ye, Y., Meyer, H.H., and Rapoport, T.A. (2001). The AAA ATPase Cdc48/p97 and its partners transport proteins from the ER into the cytosol. Nature 414, 652-656.

Zoll, S., Stanchev, S., Began, J., Skerle, J., Lepsik, M., Peclinovska, L., Majer, P., and Strisovsky, K. (2014). Substrate binding and specificity of rhomboid intramembrane protease revealed by substrate-peptide complex structures. EMBO J.

\section{Figure Legends}

\section{Figure 1. RHBDL4 contributes to efficient turnover of a soluble ERAD substrate.}

(A) Schematic representation of full-length MHC class I heavy chain (MHC-FL) and the truncated mutant MHC202 used in this study. Black box, TM domain; hexagon, site for $\mathrm{N}$ linked glycosylation; SPase, signal peptidase. Lower panel shows the crystal structure of the $\mathrm{MHC}$ ectodomain in complex with $\beta 2$-microglobulin $(\beta 2 \mathrm{~m})$ taken from the atomic coordinates 3UTQ.pdb omitting the peptide ligand in the $\alpha 1-\alpha 2$ groove. The region comprising MHC202 is highlighted in red and shown as bottom view (middle panel). Due to the C-terminal deletion of the $\alpha 3$ domain, a cluster of several hydrophobic residues is exposed in MHC202 as highlighted in orange in the surface representation of the bottom view (right panel). (B) Targeted siRNA screen identifies in addition to the Hrd1-Sel1 complex non-canonical ERAD components that contribute to MHC202 turnover. Heat map of MHC202 steady state level corresponding to Figure S1A.

(C) MHC202 is stabilized in RHBDL4 knockout cells ( $\mathrm{RHBDL} 4)$, when compared to parenteral Hek293T cells (wt). Turnover was evaluated $24 \mathrm{~h}$ post-transfection of MHC202 by adding cycloheximide ( $\mathrm{CHX}$ ) and harvesting after indicated time. Western blot (WB) quantification of full-length MHC202 is shown in the right panel (means $\pm S E M, n=3, * p \leq$ $0.05 ; * * \mathrm{p} \leq 0.01 ; * * * \mathrm{p} \leq 0.001)$. Actin was used as loading control.

(D) Hek293T cells were cotransfected with N-terminally FLAG-tagged MHC202 and either an empty vector (-), HA-tagged RHBDL4 (R4-HA) wild type (wt), or the catalytic inactive SA mutant. RHBDL4 generates an 18-kDa N-terminal cleavage fragment (open triangle) that is degraded by the proteasome as shown by increased steady state level upon MG132 treatment $(2 \mu \mathrm{M})$ compared to vehicle control (DMSO). The ectopically expressed catalytic SA mutant competes with endogenous degradation pathways for substrates and stabilizes deglycosylated full-length MHC202 (filled circle). Actin was used as loading control. 
(E) GFP-tagged RHBDL4 (R4-GFP) coimmunoprecipitates FLAG-tagged MHC202, but not FLAG-tagged OS9. Filled triangle, glycosylated MHC202, filled circle, deglycosylated MHC202. IP, immunoprecipitation.

Figure 2. RHBDL4 cleaves several but not all soluble ERAD-L substrates.

(A) Cleavage of MHC202 is specific, as RHBDL4 does not lead to fragments for Prl-KDEL and NHK even when the proteasome is inhibited by MG132 $(2 \mu \mathrm{M})$. Hek292T cells were cotransfected with N-terminally FLAG-tagged MHC202, Prolactin-KDEL or NHK with either HA-tagged RHBDL4 (R4-HA) wild-type (wt) or the SA mutant and analyzed by western blotting (WB). Filled triangle, glycosylated full-length proteins; open triangle, MHC202 cleavage product; asterisk, RHBDL4 independent NHK degradation intermediate; filled circle, deglycosylated full-length proteins.

(B) Hek293T cells were cotransfected with BACE476 $\Delta$ and either an empty vector (-), R4-HA wild type (wt), or the catalytic inactive SA mutant. RHBDL4 generates an N-terminal 40-kDa cleavage fragment (open triangle) that is degraded by the proteasome as shown by increased steady state level upon MG132 treatment $(2 \mu \mathrm{M})$ compared to vehicle control (DMSO). In presence of MG132 $(2 \mu \mathrm{M})$ the 34-kDa deglycosylated full-length BACE476 $\triangle$ (filled circle) and traces of a deglycosylated form of the RHBDL4-generated cleavage fragment (open circle) become visible.

(C) Cleavage assay as in (B), but with N-terminally FLAG-tagged RI332 as substrates generating cleavage fragments in the range of 25 to $35 \mathrm{kDa}$ (open triangles). Filled circle, deglycosylated full-length RI332.

\section{Figure 3. RHBDL4 cleaves MHC202 in order to facilitate p97-dependent dislocation of} cleavage products.

(A) RHBDL4 cleaves C-terminally FLAG-tagged MHC202 with an additional C-terminal glycosylation site (K197N) post translocational as shown by sensitivity of the C-terminal fragment to Endo $\mathrm{H}$ (open triangle and open circle). R4-HA, HA-tagged RHBDL4; hexagon, site for N-linked glycosylation; SPase, signal peptidase.

(B) Clearance of RHBDL4 generated cleavage product depends on p97, as p97 inhibitor CB$5083(2.5 \mu \mathrm{M})$ stabilized the N-terminal MHC202 fragment (open triangle) even in absence of proteasome inhibitor MG132 $(2 \mu \mathrm{M})$. Likewise, CB-5083 reduced appearance of the deglycosylated unprocessed form of MHC202 (filled circle) that is observed upon MG132 treatment, confirming that also the Hrd1-dependent dislocation pathway depends on p97.

\section{Figure 4. Processing by RHBDL4 is determined by specific features and not general} substrate ubiquitination. 
(A) Outline of MHC202, MHC-FL and a chimera of MHC and pT $\alpha$ (indicated in blue). SPase, signal peptidase.

(B) Hek293T cells were cotransfected with N-terminally FLAG-tagged MHC202 or MHC-FL and either HA-tagged RHBDL4 (R4-HA) wild-type (wt) or SA mutant, as well as Myc-tagged US11. Proteasome inhibitor MG132 (2 $\mu \mathrm{M})$ was added. Open triangle, N-terminal MHC202 fragment; filled circle, deglycosylated form of MHC202; asterisk, RHBDL4 independent degradation intermediate. Actin was used as loading control.

(C) Fusion to the pT $\alpha$ TM degron renders MHC susceptible for RHBDL4 cleavage in cellbased assay as in (B).

(D) RHBDL4 cleavage assays for MHC202 deletion and point mutants were performed. Proteasome inhibitor MG132 (2 $\mu \mathrm{M})$ was added. Cleavage by RHBDL4 occurred between amino acid 121 and amino acid 128. Small amino acids within this stretch were mutated to phenylalanine $(F)$. Open triangle, $\mathrm{N}$-terminal fragment; filled circle, deglycosylated full-length MHC202. Right panel shows the position of the two critical glycine residues (G121 and $\mathrm{G} 125$ ) and the 121-128 cleavage site region (red) in the MHC202 structure model as shown in Figure $1 \mathrm{~A}$.

(E) Cleavage assay as in (D) but with MHC202 proline point mutants. Open triangle, Nterminal fragment; filled circle, deglycosylated MHC202.

\section{Figure 5. The erlin ERAD complex interacts with RHBDL4 and MHC202.}

(A) SILAC-based mass spectrometry analysis of RHBDL4 interactome from Triton X-100solubilized Hek293T cells with chromosomally FLAG-tagged RHBDL4 (Hek293T R4-FLAG) as outlined. Right panel shows volcano plot representation of RHBDL4 interaction partners identified in all three replicates.

(B) Microsome fraction of Hek293T cells was solubilized with $1 \%$ Triton X-100 and endogenous RHBDL4 was isolated by immunoprecipitation (IP). Western blotting (WB) identifies copurification of endogenous Erlin2. CLIMP63 was used as negative control. (C) Endogenous Erlin2 interacts more pronounced with GFP-tagged RHBDL4 (R4-GFP) wild type (wt) than with its catalytic inactive SA mutant. Derlin1 was used as negative control. (D) RHBDL4 is part of a MDa-sized erlin complex. Hek293T cells transfected with empty vector (-) or FLAG-tagged RHBDL4 (R4-FLAG), respectively, were solubilized with 1\% Triton X-100, immunoprecipitated for FLAG, eluted with FLAG peptides and analyzed by BN-PAGE. RHBDL4-FLAG formed several higher molecular weight complexes in addition to the 1.2 MDa complex containing Erlin2.

(E) Erlin1-HA (1) and Erlin2-HA (2) specifically interacted with MHC202 but show no interaction to Prl. Hc, heavy chain; Ic, light chain; asterisk, unspecific band. 
(F) MHC202 is stabilized in Erlin2 Hek293T knockout cells ( $\Delta$ Erlin2) when compared to parental Hek293T cells (wt), as shown by cycloheximide ( $\mathrm{CHX}$ ) chase. Actin used as loading control. Lower panel, western blot quantification of MHC202 (means $\pm \mathrm{SEM}, \mathrm{n}=4, * * * \mathrm{p} \leq$ $0.001)$.

Figure 6. RHBDL4 reduces burden of aggregation-prone ERAD-L substrates.

(A) The aggregation-prone model protein ER $\beta$ harboring an $\mathrm{N}$-terminal Myc tag interacts with the catalytic SA mutant of GFP-tagged RHBDL4 (R4-GFP) as shown by immunoprecipitation (IP), whereas no interaction is observed for the wild-type (wt) construct. WB, western blotting.

(B) ER $\beta$ harboring an N-terminal Myc-tag and a C-terminal FLAG-tag was co-expressed with HA-tagged RHBDL4 (R4-HA) as indicated. Tris-bicine-urea SDS PAGE and western blot (WB) analysis reveals at least two C-terminal cleavage fragments along full-length $E R \beta$ and an undetermined post-translational modification (star).

(C) A mutation in fibrinogen $\alpha$-chain (R375W) that increased propensity for aggregation also increased generation of two $\mathrm{N}$-terminal fragments (open arrows) by ectopically expressed HA-tagged RHBDL4 (R4-HA). Actin was used as loading control.

(D) MHC202 steady state levels were analyzed in Hek293T cells transfected with two independent shRNAs targeting RHBDL4 (R4-1 and R4-2) or non-targeting control (nt) by NP40 lysis followed by western blot analysis of the soluble and detergent-insoluble fraction. p97 was used as loading control for soluble fraction and H2B for insoluble fraction, respectively.

Figure 7. Model for RHBDL4-erlin-mediated clearance of pre-aggregates.

Monomeric ERAD-L substrates are predominantly degraded through the canonical Hrd1-Sel1 dislocation pathway whereas the erlin complex targets aggregation-prone conformations. RHBDL4-catalyzed clipping facilitates dislocation of cleavage fragments by an unknown mechanism. Upon increase of their concentration, pre-aggregation species form oligomers that may be subject for a putative erlin disaggregase function. Large, macroscopic aggregates cannot be targeted to the ERAD pathway and become subject for lysosomal degradation routes (not shown). 


\section{Supplemental Material}

Figure S1. MHC202 is degraded by a concerted action of the Hrd1-Sel1 complex and RHBDL4. Related to Figure 1.

(A) Influence of selected ERAD components on steady-state level of MHC202 shown as western blot of lysates from siRNA transfected cells detecting the C-terminal FLAG tag. p97 was used as positive and loading control.

(B) RHBDL4 knockdown with two independent shRNAs (R4-1 and R4-2) in Hek293T cells leads to increase of MHC202 steady state level, when compared to a non-targeting (nt) control shRNA. Actin was used as loading control.

(C) Sanger sequencing of chromosomal DNA obtained from the TALEN-based Hek293T RHBDL4 knockout cell line (referred to also by its gene name Rhbdd1). TALEN target site is underlined.

(D) Simultaneous knockdown of RHBDL4 (R4) and Hrd1 further increases MHC202 steady state level when compared to single knockdown or non-targeting (nt) control as shown by WB analysis (means $\pm \mathrm{SEM}, \mathrm{n}=5, * \mathrm{p} \leq 0.05 ; * * \mathrm{p} \leq 0.01$ ). Actin was used as loading control; nt, non-targeting control siRNA.

(E) MHC202 and its $18 \mathrm{kDa}$-terminal fragment generated by ectopically expressed HAtagged RHBDL4 (R4-HA) are glycosylated as shown by sensitivity to Endo $H(H)$ and PNGase (P). RHBDL4 gain-of-function assay as shown in Figure 1E either treated with vehicle (DMSO) or MG132 treatment $(2 \mu \mathrm{M})$. WB, western blotting.

Figure S2. RHBDL4-catalyzed cleavage of BACE476 $\Delta$ generates glycosylated Nterminal fragment. Related to Figure 2.

(A) BACE476 $\triangle$ (filled triangle) and its RHBD4-generated N-terminal fragment (open triangle) are glycosylated as shown by sensitivity to Endo $\mathrm{H}$ (filled and open circles). WB, western blotting.

(B) Hek293T cells were cotransfected either with RI332 or RPN1 and an empty vector (-), HA-tagged RHBDL4 (R4-HA) wild type (wt), or the catalytic inactive SA mutant. RHBDL4 generates several $\mathrm{N}$-terminal cleavage fragments. Expression of the catalytic mutant stabilizes the 40-kDa deglycosylated full-length RI332 (filled circle) even in absence of the proteasome inhibitor MG132.

Figure S3. RHBDL4 knockdown leads to accumulation of MHC202 in the ER. Related to Figure 3.

(A) RHBDL4 cleaves MHC202 lacking the native glycosylation site (N100Q) with a single glycosylation site (K197N) in the C-terminal portion leading to an Endo $\mathrm{H}$-sensitive and a 
partially deglycosylated fragment (open triangle and open circle). Hexagon, site for $\mathrm{N}$-linked glycosylation; SPase, signal peptidase; WB, western blotting.

(B) Knockdown of RHBDL4 with two independent shRNAs (R4-1, R4-2) leads to MHC202 accumulation in the ER as shown by colocalization with RFP-KDEL; nt, non-targeting control shRNA; scale bar, $10 \mu \mathrm{M}$.

(C) Knockdown of RHBDL4 with two independent shRNAs (R4-1, R4-2) increases MHC202 signal in stable T-REx Hek293T cell expressing FLAG-tagged MHC202, when compared to non-targeting (nt) control shRNA; scale bar, $10 \mu \mathrm{M}$.

\section{Figure S4. US11 increases turnover of MHC-FL. Related to Figure 4.}

Hek293T cells were transfected with MHC-FL with or without Myc-tagged US11. 24 h posttransfection cycloheximide was added and cells harvested at indicated timepoints (means \pm SEM, $\mathrm{n}=4 ; * * * * \mathrm{p} \leq 0.0001)$.

Figure S5. RHBDL4 interacts with Erlin1, Erlin2 and RNF170. Related to Figure 5.

(A) Outline of the applied tagging strategy of RHBDL4 (referred to also by its gene name Rhbdd1) according to (Fueller et al., 2019).

(B) Sanger sequencing of chromosomal DNA obtained from the Hek293T-R4-FLAG cell lines shows insertion of the FLAG tag in the last coding exon. Color code as in (A).

(C-E) Hek293T cells were transfected with Erlin1-FLAG, Erlin2-FLAG or RNF170-FLAG in addition to empty vector or RHBDL4-GFP. Following solubilization with Triton X-100, RHBDL4-GFP was isolated by immunoprecipitation (IP) using an anti-GFP antibody. Western blot (WB) reveals co-purification of RHBDL4 binding partners.

(F) Microsome fraction of Hek293T cells was solubilized with $1 \%$ Triton X-100 and endogenous Erlin2 was isolated by immunoprecipitation (IP). Western blotting identifies copurification of endogenous RHBDL4.

(G) Endogenous RHBDL4 is part of a MDa-sized erlin complex. Parenteral Hek293T cells (wt) or cells with a chromosomally FLAG-tagged RHBDL4 (FLAG), respectively, were solubilized with $1 \%$ Triton X-100, immunoprecipitated for FLAG, eluted with FLAG peptides and analyzed by BN-PAGE. RHBDL4-FLAG formed several higher molecular weight complexes in addition to the 1.2 MDa complex containing Erlin2. WB, western blotting. (H) Sanger sequencing of chromosomal DNA obtained from the CRISPR/Cas9-based Hek293T Erlin2 knockout cell line. Single-guide RNA-binding site is underlined; protospacerassociated motif is shown in bold. 
Figure S6. Erlin2 interacts with ERß. Related to Figure 6.

(A) Western blotting (WB) after immunoprecipitation (IP) of Erlin2-HA from Triton X-100 solubilized Hek293T cells confirms interaction to ER $\beta$ as has been shown (Vincenz-Donnelly et al., 2018).

(B) Efficiency of RHBDL4-catalyzed cleavage of $\gamma$-fibrinogen-R375W by ectopically expressed HA-tagged RHBDL4 as show in Figure 6B normalized to wild type (wt) fibrinogen $\gamma$-chain (means \pm SEM, $n=3$ ).

Table S1. Complete list of proteins identified by shotgun proteomics on immunoisolated endogenously FLAG-tagged RHBDL4.

See separate file. 


\section{Kühnle et al. Figure 1}

A
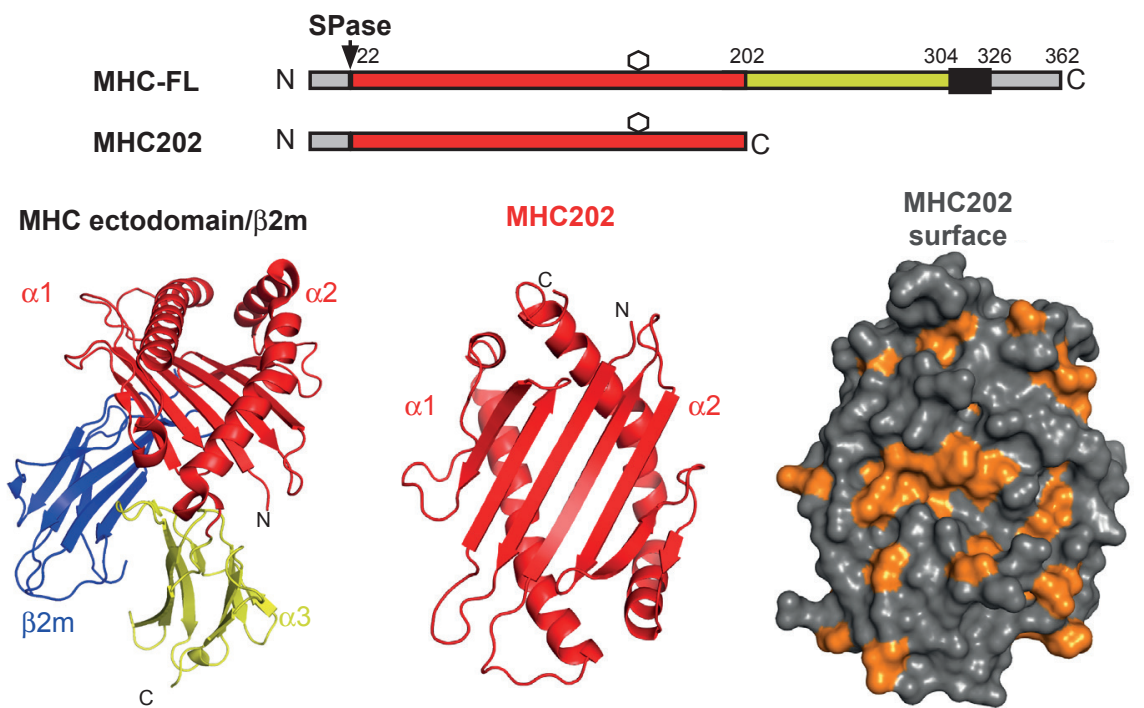

B

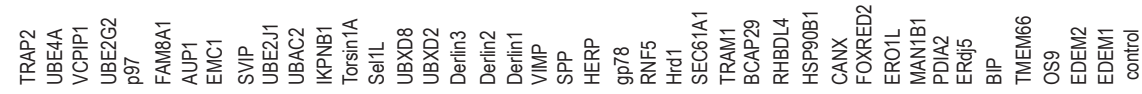

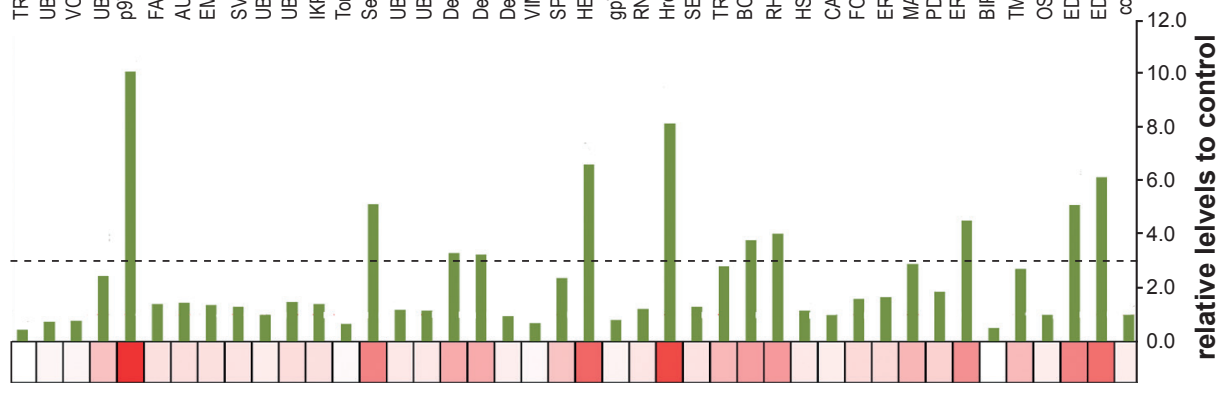

C
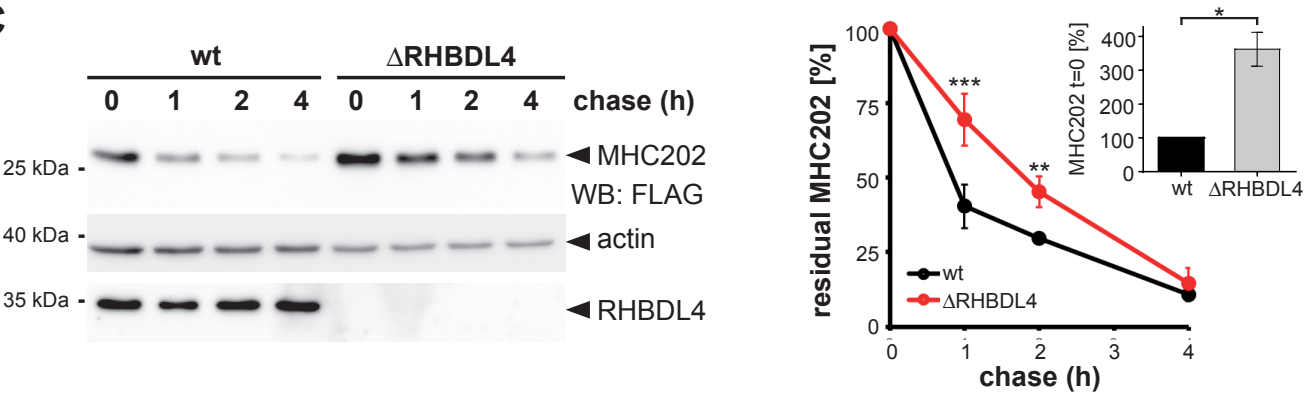

D

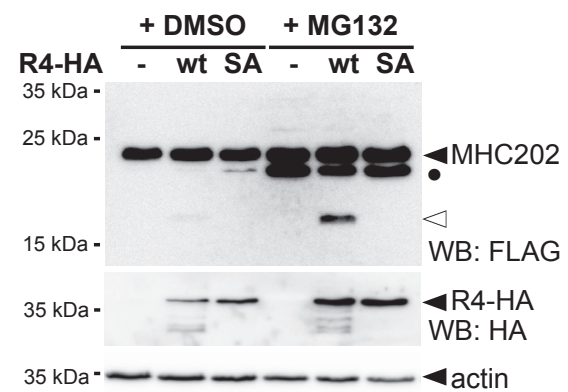

E

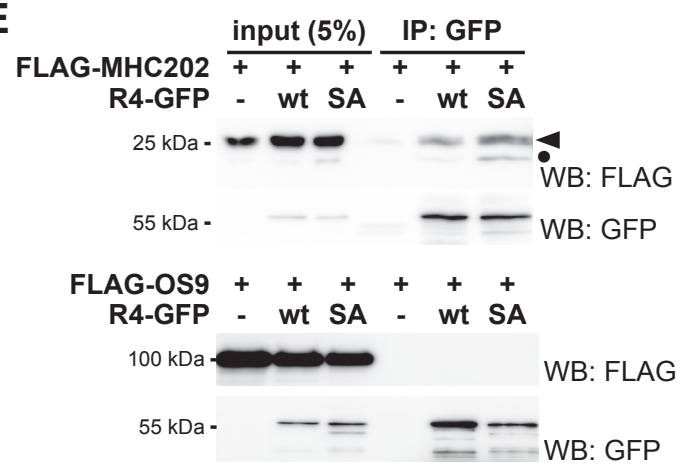




\section{Kühnle et al. Figure 2}

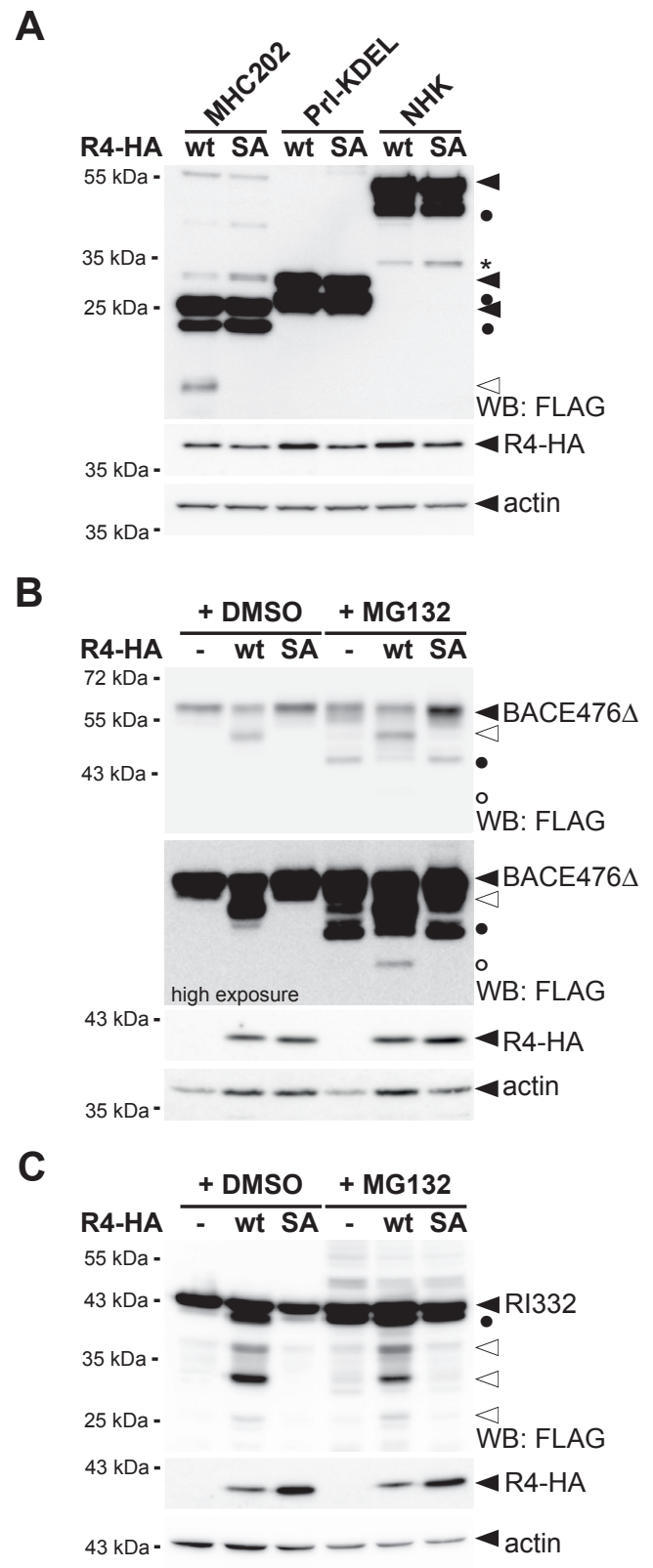


bioRxiv preprint doi: https://doi.org/10.1101/848754; this version posted November 20, 2019. The copyright holder for this preprint (which was not certified by peer review) is the author/funder. All rights reserved. No reuse allowed without permission.

\section{Kühnle et al. Figure 3}

A

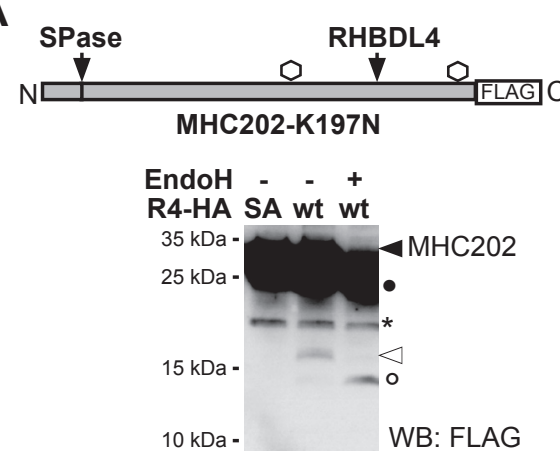

B

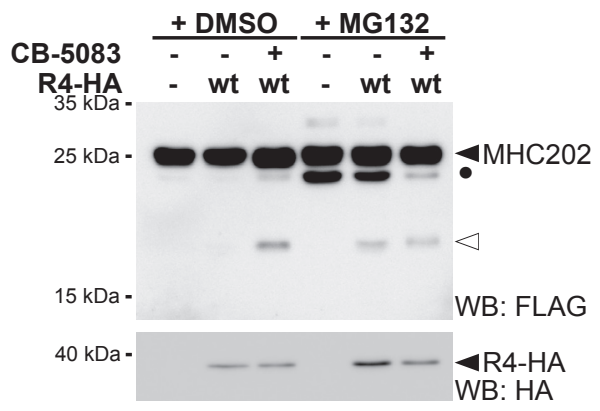




\section{Kühnle et al. Figure 4}

A

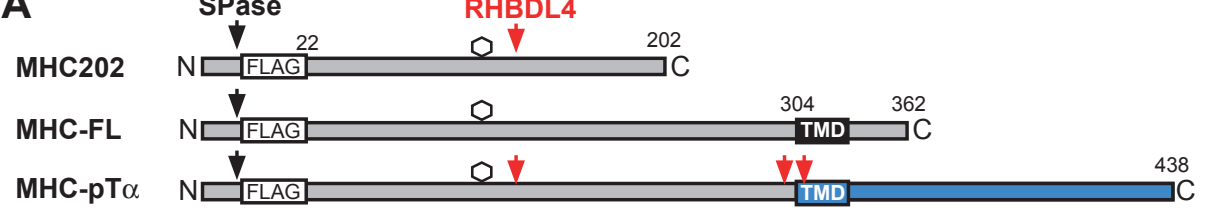

B

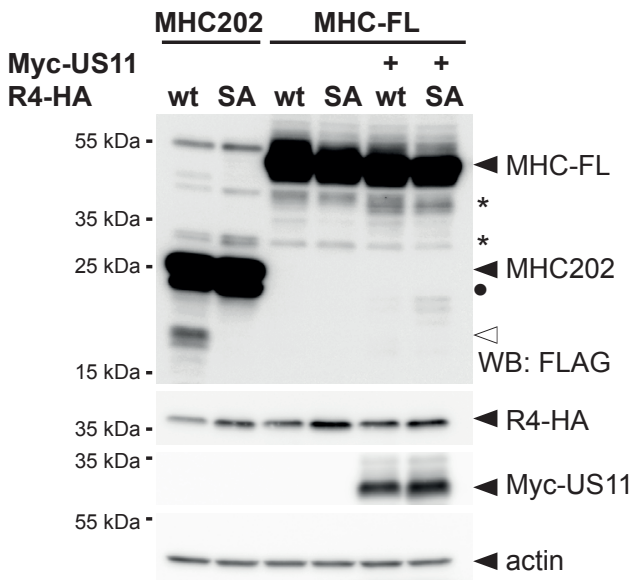

C

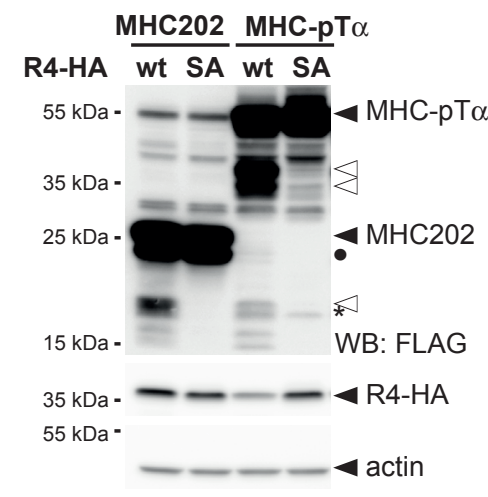

D

$\begin{array}{ll}\text { wt } & { }^{114} \\ \Delta \text { 121-128 } & \text { HTVQRMYGCDVGSDWRF } \\ \text { 121FF } & \text { HTVQRMY-------RF } \\ \text { 125FF } & \text { HTVQRMYFFDVGSDWRF } \\ \text { 121FF125FF } & \text { HTVQRMYGCDVFFDWRF } \\ & \text { HTVQRMYFFDVFFDWRF }\end{array}$
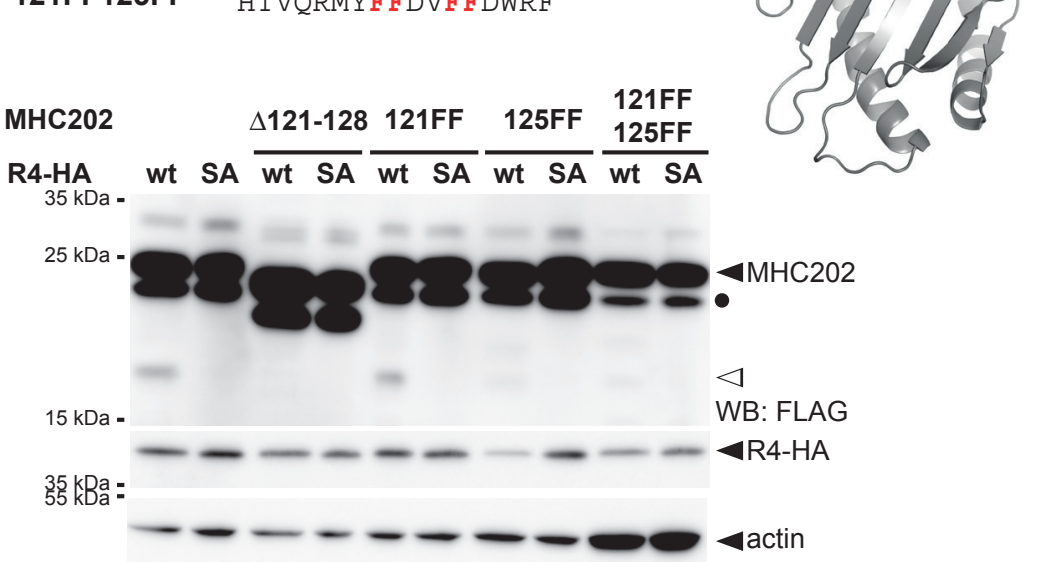

E

114

YGCDVGSDWRF HTVQRMYPCDVGSDWRF HTVQRMYGPDVGSDWRF

PP HTVQRMYPCDVGPDWRF
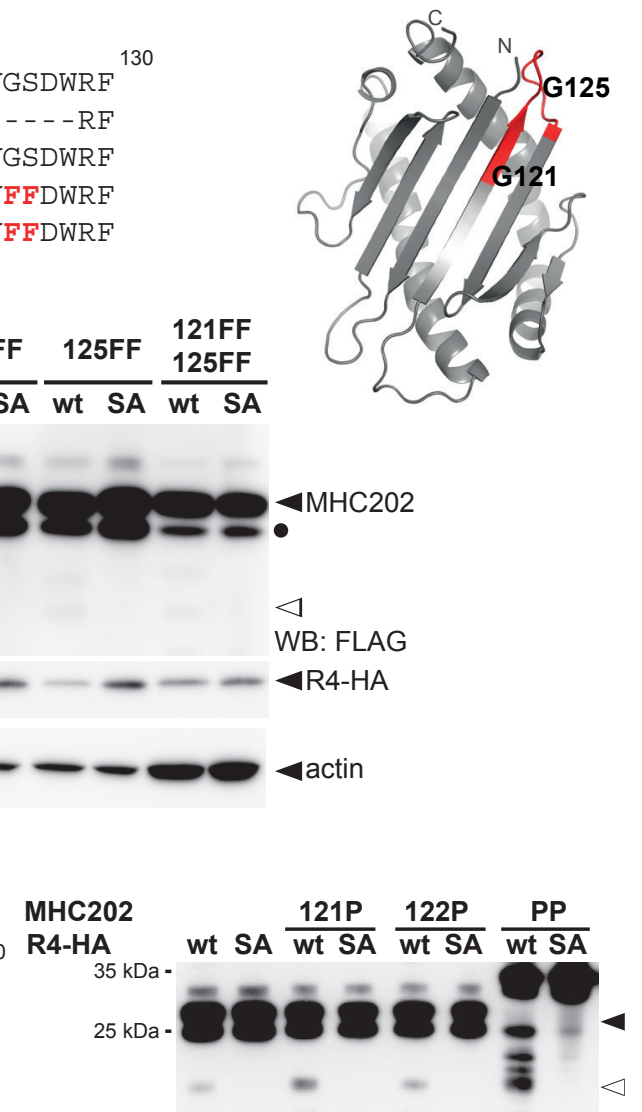


\section{Kühnle et al. Figure 5}

A

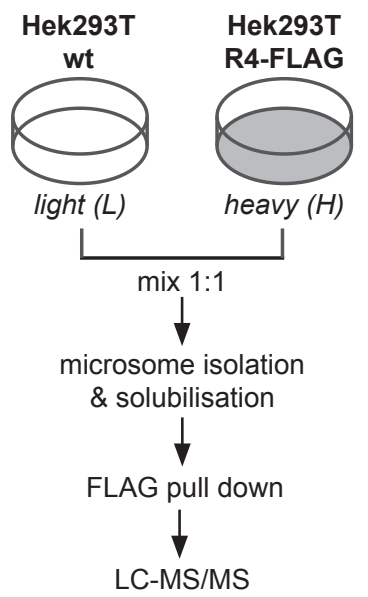

B

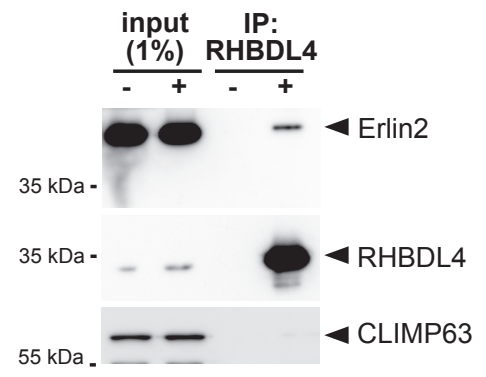

D

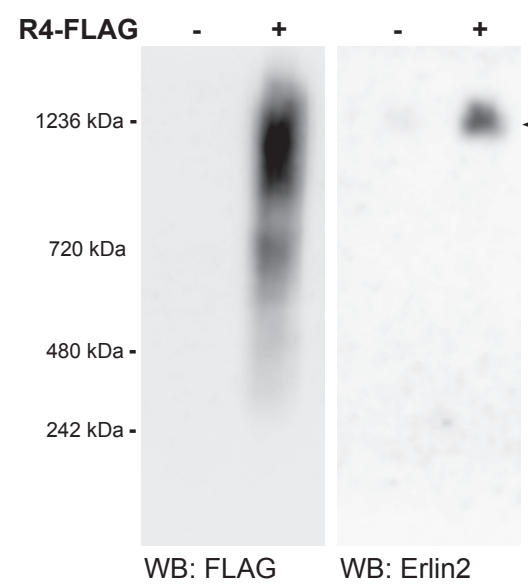

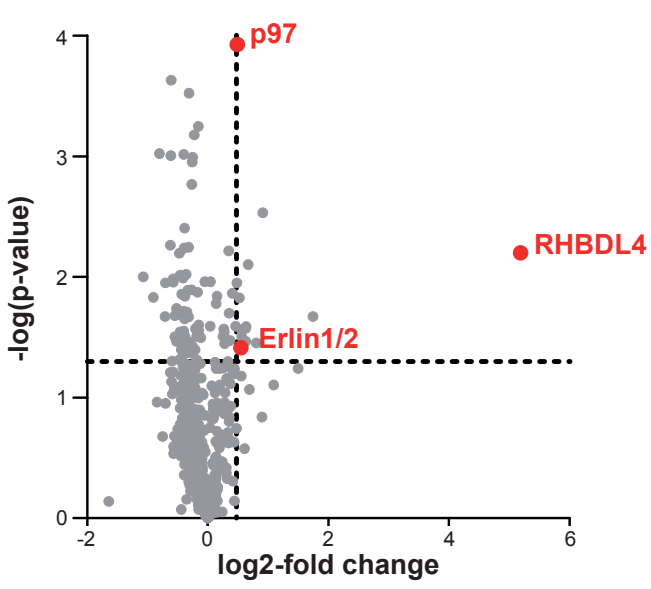

C

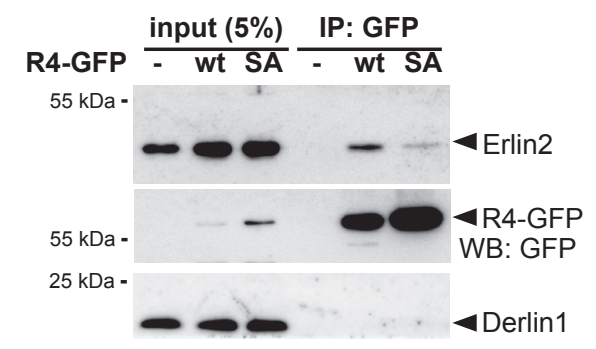

$\mathbf{F}$
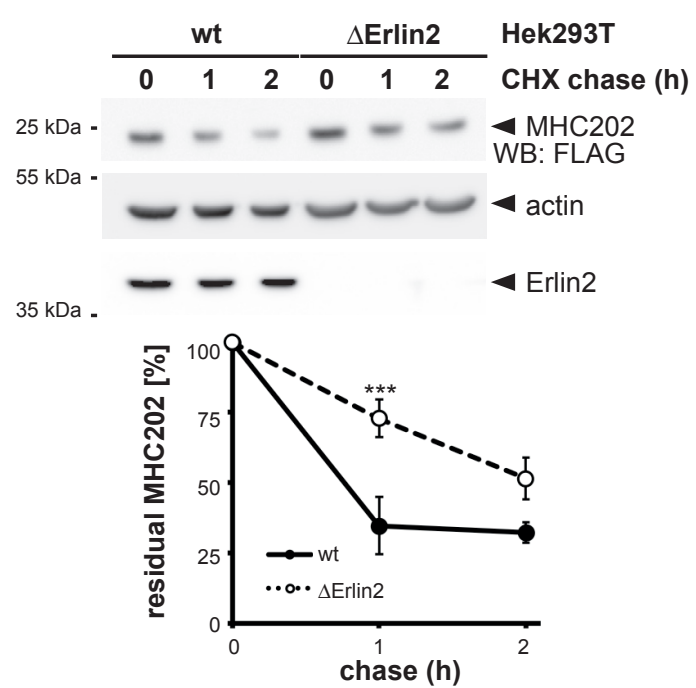

E
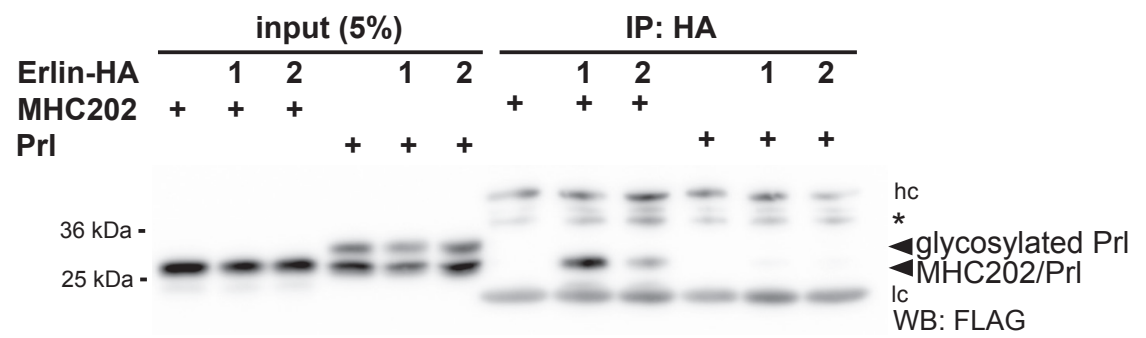

$55 \mathrm{kDa}$ - 


\section{Kühnle et al. Figure 6}

A

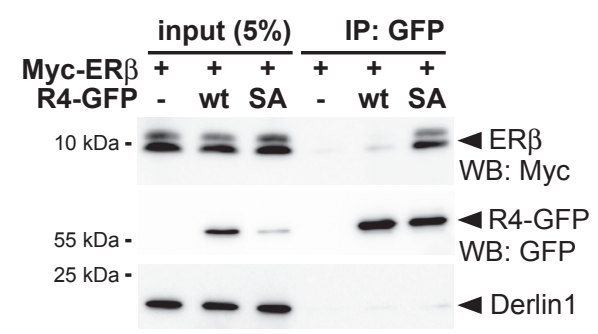

C $\gamma$-fibrinogen wt R375W

R4-HA - SA wt wt SA -

$35 \mathrm{kDa}$ -

๑OCOOC $\gamma$-fibrinogen

$25 \mathrm{kDa}-$

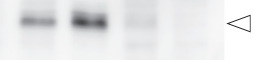

$\triangleleft$

WB: FLAG

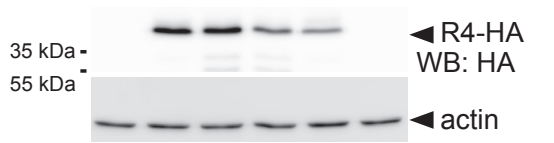

B

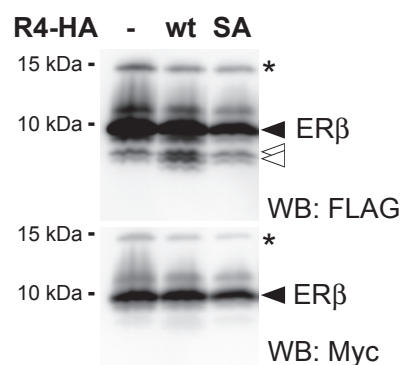

D

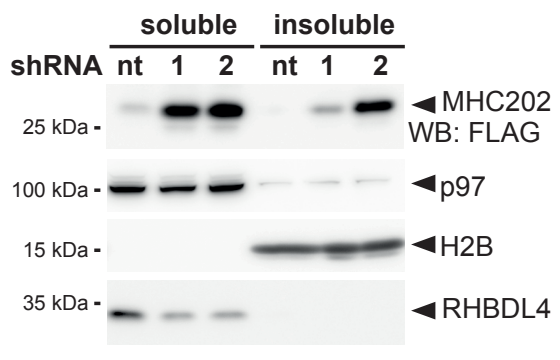


bioRxiv preprint doi: https://doi.org/10.1101/848754; this version posted November 20, 2019. The copyright holder for this preprint (which was not certified by peer review) is the author/funder. All rights reserved. No reuse allowed without permission.

Kühnle et al. Figure 7

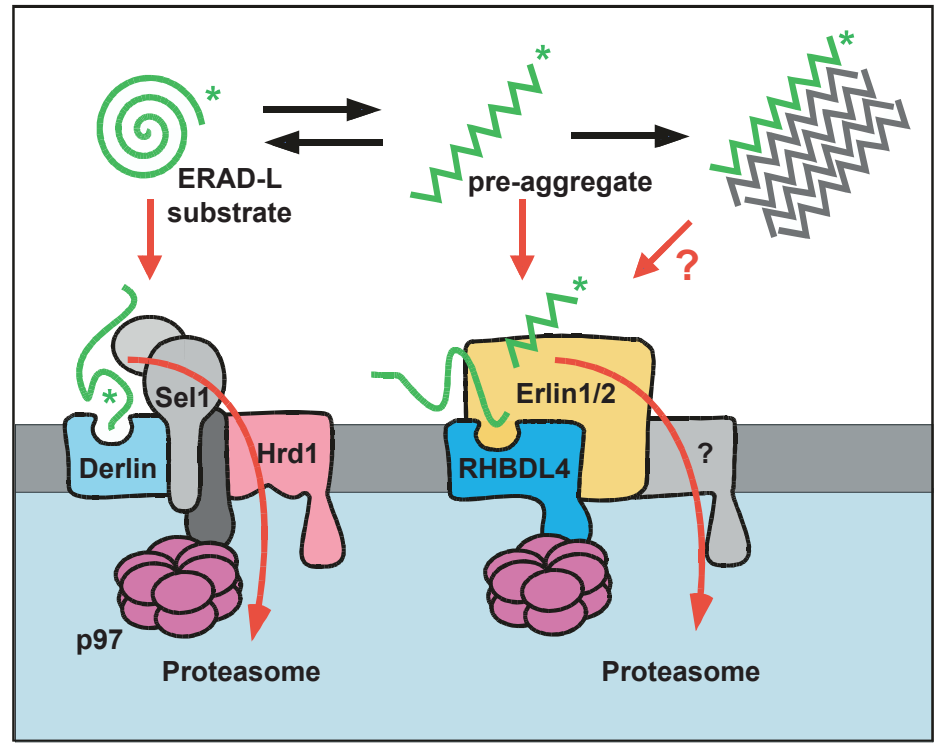




\section{Kühnle et al. Figure S1}

A

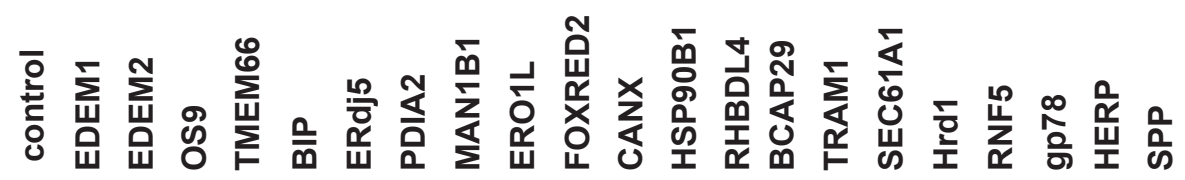

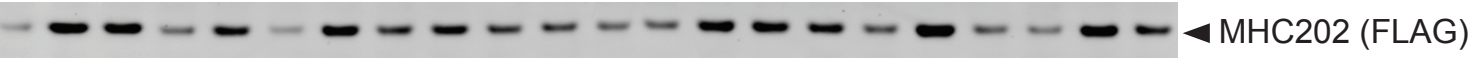

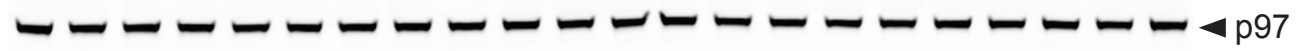

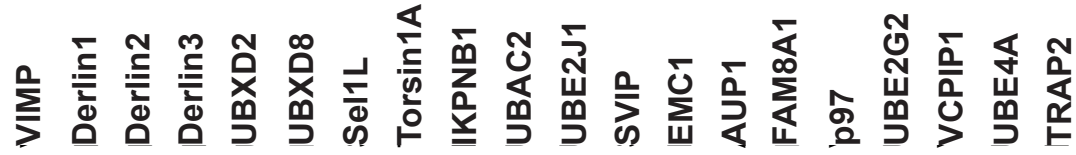

- - - - - - - - - - - - MHC202 (FLAG)

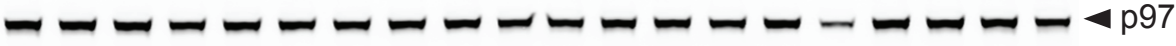

B

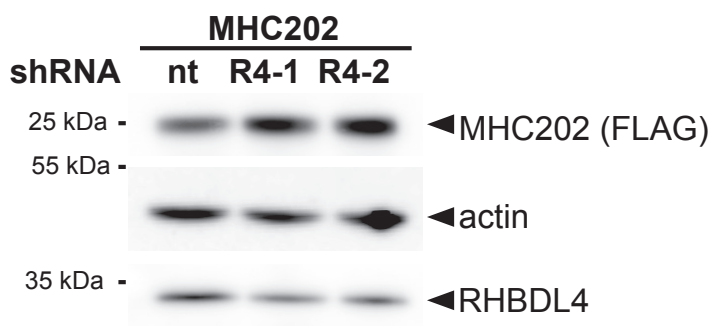

C

RHBDL4/Rhbdd1 chromosome 2, exon 4

wt CATTTGTATTTCAATATGGCATCCATGCTCTGGAAAGGAATAAATCTAGAAAGAAG/GTCATTTTATGAAGTGTGGGTCCCTAT allele1 CATTTGTATTTCAATATGG - ........... AAAGGAATAAATCTAGAAAGAAG/GTCATTTTATGAAGTGTGGGTCCCTAT allele2 CATTTGTATTTCAATATGGCAT-.............................. / - AAATCTAGAAGTGTGGGTCCCTAT

D
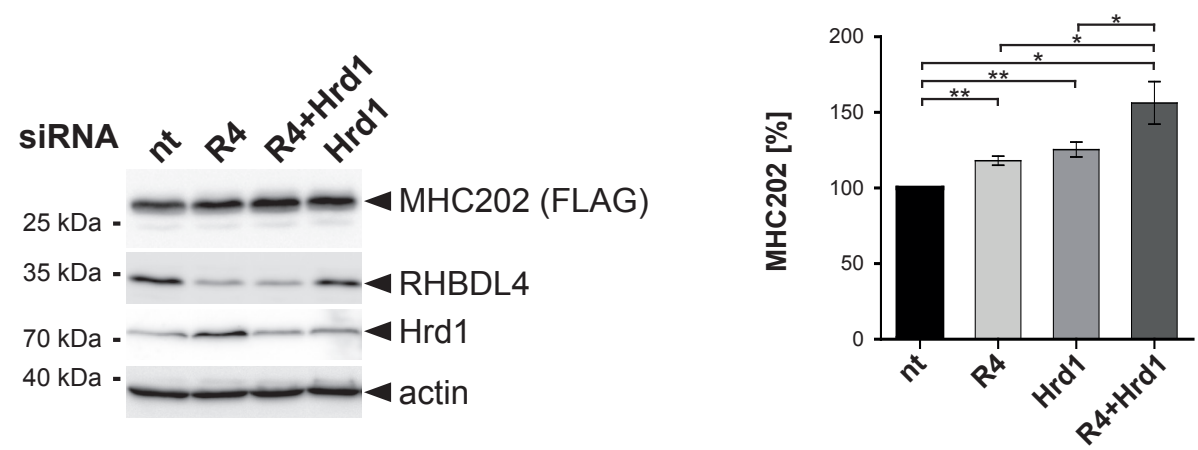

E

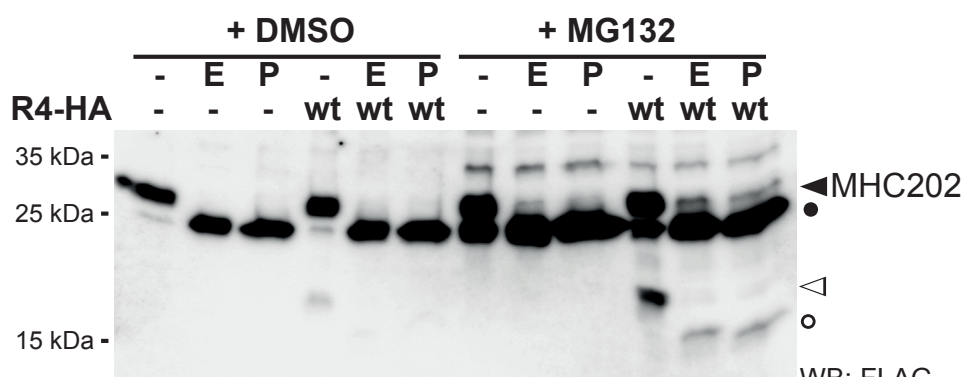


bioRxiv preprint doi: https://doi.org/10.1101/848754; this version posted November 20, 2019. The copyright holder for this preprint (which was not certified by peer review) is the author/funder. All rights reserved. No reuse allowed without permission.

\section{Kühnle et al. Figure S2}

A

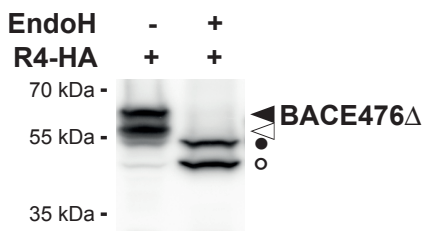

$35 \mathrm{kDa}$ -

WB: FLAG

B

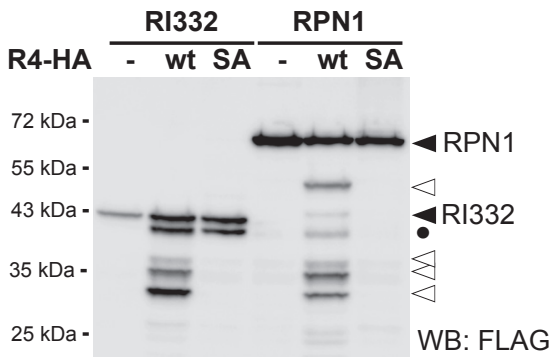




\section{Kühnle et al. Figure S3}

A

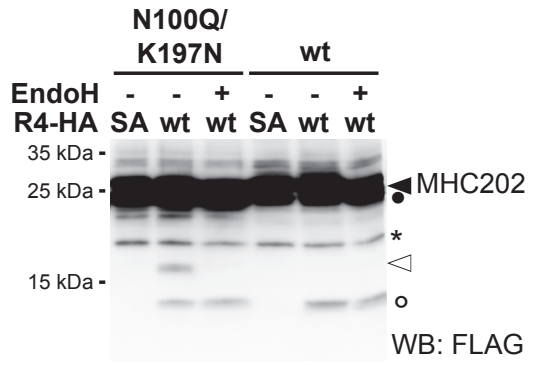

B

ShRNA

- MHC202

- RFP-KDEL

merge

nt
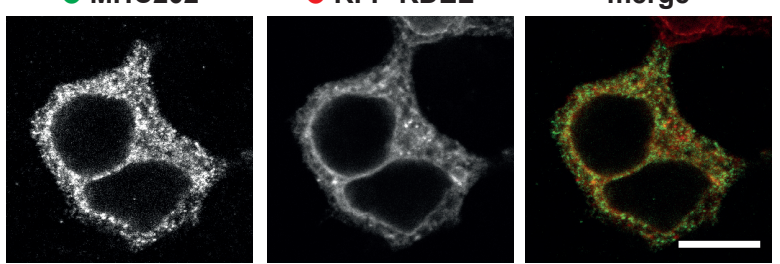

R4-1
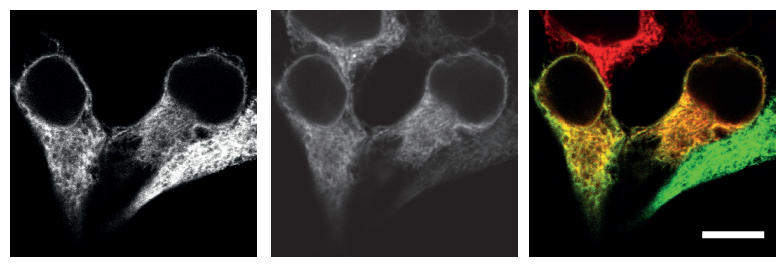

R4-2
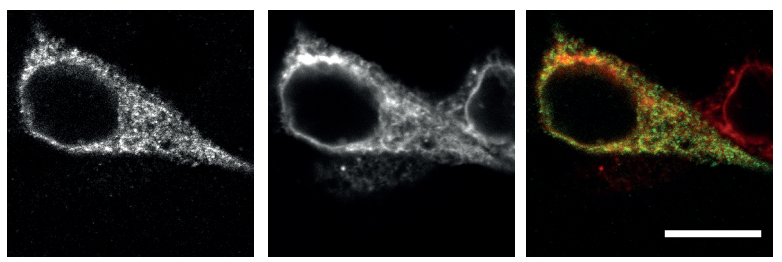

C

shRNA

RHBDL4

- MHC202

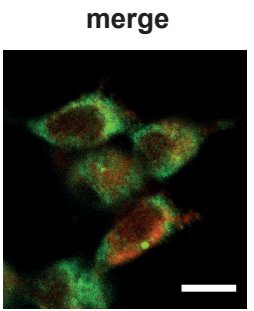

R4-1
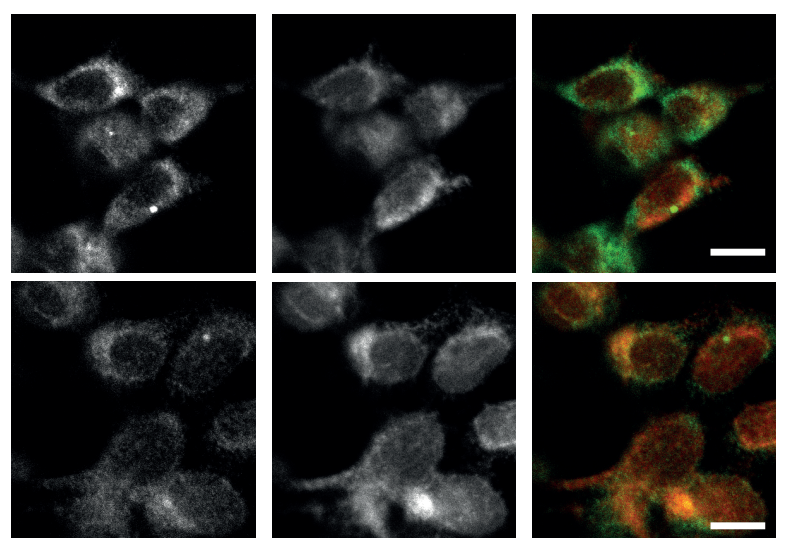

R4-2
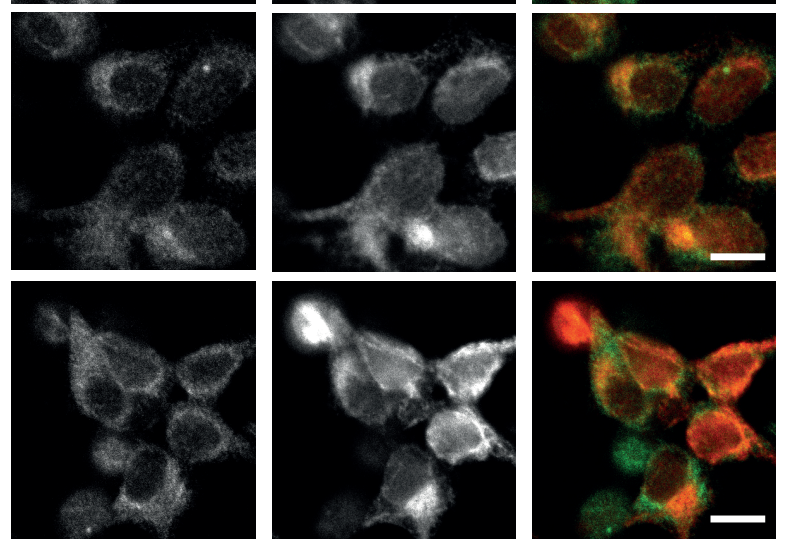
bioRxiv preprint doi: https://doi.org/10.1101/848754; this version posted November 20, 2019. The copyright holder for this preprint (which was not certified by peer review) is the author/funder. All rights reserved. No reuse allowed without permission.

\section{Kühnle et al. Figure S4}
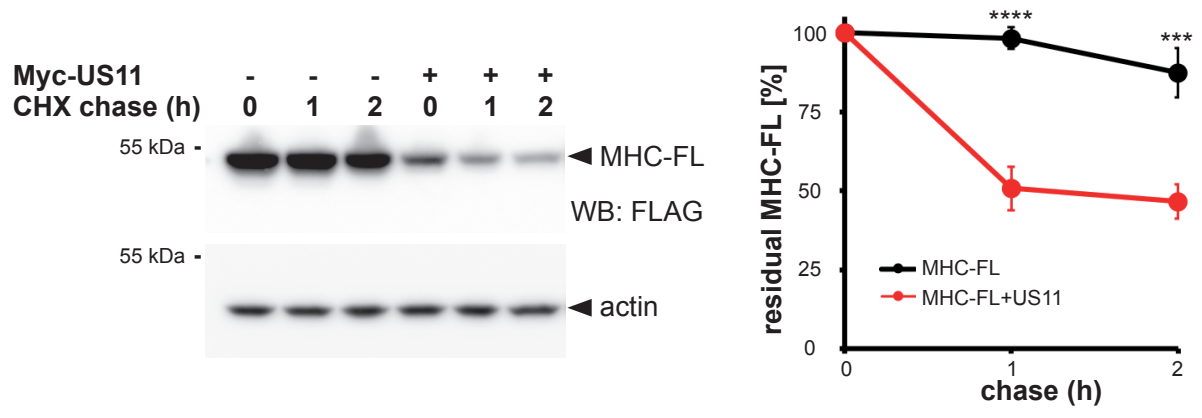
bioRxiv preprint doi: https://doi.org/10.1101/848754; this version posted November 20, 2019. The copyright holder for this preprint (which was not certified by peer review) is the author/funder. All rights reserved. No reuse allowed without permission.

\section{Kühnle et al. Figure S5}

A

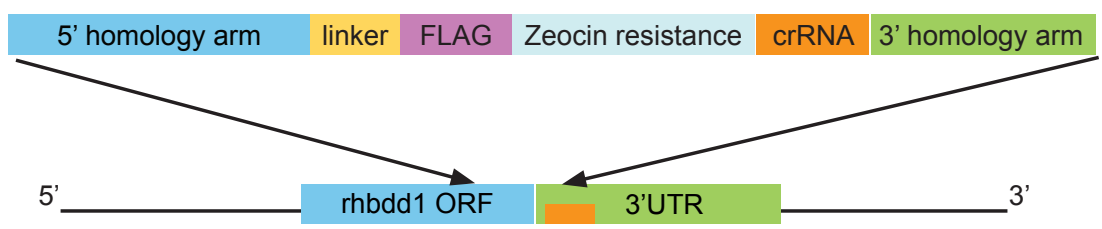

B

RHBDL4/Rhbdd1 chromosom 2 exon 7

$\begin{array}{llllllllllllllllllllllllll}G & N & T & R & N & S & P & P & P & Y & G & F & H & L & S & P & E & E & M & R\end{array}$

GGAAATACCAGAAATAGCCCACCACCCTACGGGTTTCATCTCTCACCAGAAGAAATGAGG

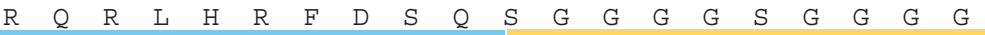

AGACAGCGGCTTCACAGATTCGATAGCCAGTCAGGTGGAGGAGGTAGTGGCGGAGGCGGA

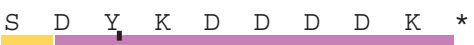

TCCGATTACAAGGATGACGATGACAAGTAG

C

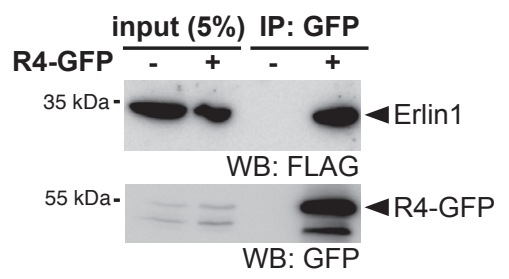

D

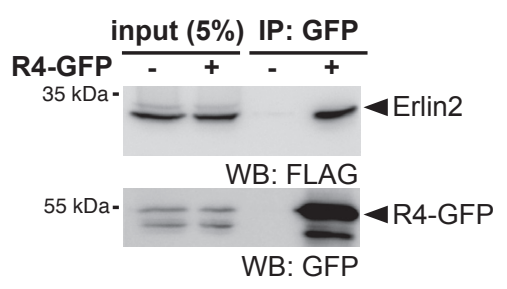

$\mathbf{E}$

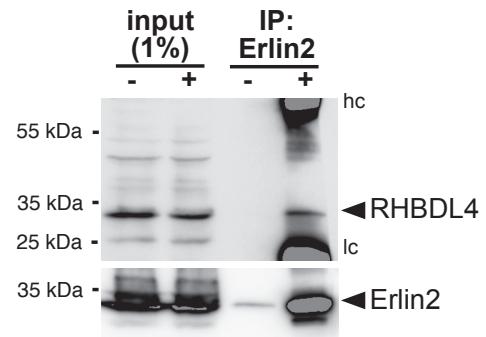

$\mathbf{F}$

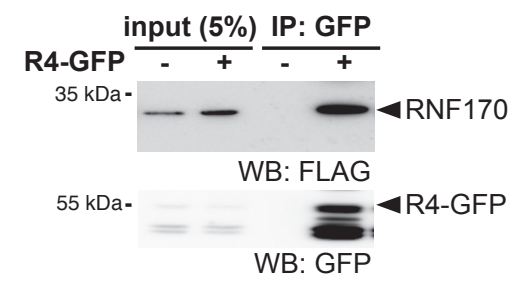

G

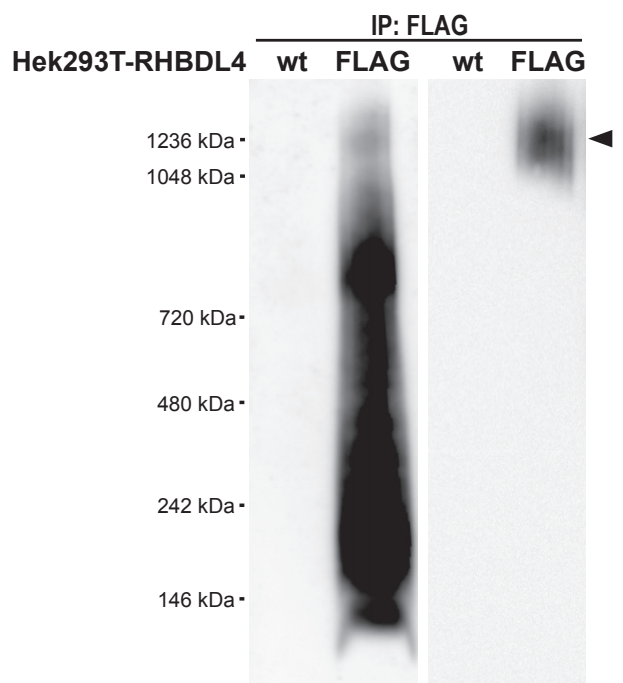

WB: RHBDL4 WB: Erlin2

H

Erlin2 chromosome 8, exon 2

wt AGTTTCTTTTGTGCATCTCTCTTCTCAGCTGTGCACAAGATAGAAGAGGGACATATTGGGGTATATTACAGGTAAGGCAGAGAC allele 1 AGTTTCTTTTGTGCATCTCTCTTCTCAGCTGTGCACAAGAT- -..--GGACATATTGGGGTATATTACAGGTAAGGCAGAGAC allele 2 AGTTTCTTTTGTGCATCTCTCTTCTCAGCTGTGCACAAGATAG---_GGGACATATTGGGGTATATTACAGGTAAGGCAGAGAC allele 3 AGTTTCTTTTGTGCATCTCTCTTCTCAGCTGTGCACAAGATAGA - . . . . CATATTGGGGTATATTACAGGTAAGGCAGAGAC 
bioRxiv preprint doi: https://doi.org/10.1101/848754; this version posted November 20, 2019. The copyright holder for this preprint (which was not certified by peer review) is the author/funder. All rights reserved. No reuse allowed without permission.

\section{Kühnle et al. Figure S6}

A

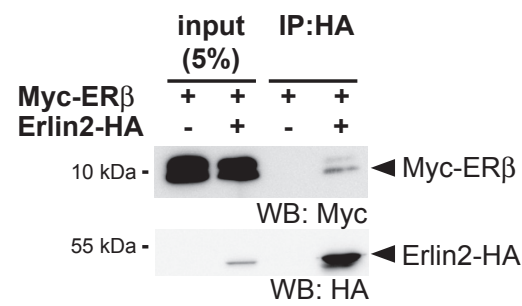

B

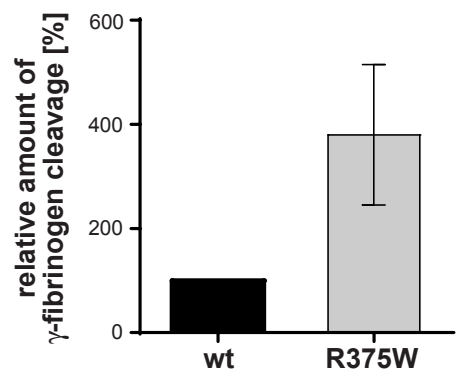

